\title{
Mini Review: Advances in 2-Haloacid Dehalogenases
}

\author{
Yayue Wang ${ }^{1}$, Qiao Xiang ${ }^{1,2}$, Qingfeng Zhou ${ }^{1}$, Jingliang $X u^{3,4 *}$ and Dongli Pei ${ }^{1 *}$ \\ ${ }^{1}$ College of Biology and Food, Shangqiu Normal University, Shangqiu, China, ${ }^{2}$ College of Life Sciences, Henan Normal \\ University, Xinxiang, China, ${ }^{3}$ School of Chemical Engineering, Zhengzhou University, Zhengzhou, China, ${ }^{4}$ Zhengzhou \\ Tuoyang Industrial Co., Ltd., Zhengzhou, China
}

\section{OPEN ACCESS}

Edited by:

Benwei Zhu,

Nanjing Tech University, China

Reviewed by:

Fahrul Huyop

Universiti Teknologi Malaysia, Malaysia

Dirk Tischler,

Ruhr University Bochum, Germany

${ }^{*}$ Correspondence:

Jingliang $X u$

xujl@zzu.edu.cn

Dongli Pei

peidongli@126.com

Specialty section:

This article was submitted to

Microbiotechnology,

a section of the journal

Frontiers in Microbiology

Received: 15 August 2021

Accepted: 13 September 2021

Published: 15 October 2021

Citation:

Wang Y, Xiang Q, Zhou Q, Xu J and Pei D (2021) Mini Review: Advances in

2-Haloacid Dehalogenases.

Front. Microbiol. 12:758886.

doi: 10.3389/fmicb.2021.758886
The 2-haloacid dehalogenases (EC 3.8.1.X) are industrially important enzymes that catalyze the cleavage of carbon-halogen bonds in 2-haloalkanoic acids, releasing halogen ions and producing corresponding 2-hydroxyl acids. These enzymes are of particular interest in environmental remediation and environmentally friendly synthesis of optically pure chiral compounds due to their ability to degrade a wide range of halogenated compounds with astonishing efficiency for enantiomer resolution. The 2-haloacid dehalogenases have been extensively studied with regard to their biochemical characterization, protein crystal structures, and catalytic mechanisms. This paper comprehensively reviews the source of isolation, classification, protein structures, reaction mechanisms, biochemical properties, and application of 2-haloacid dehalogenases; current trends and avenues for further development have also been included.

Keywords: 2-haloacid dehalogenases, protein structure, catalytic mechanism, biochemical properties, application

\section{INTRODUCTION}

Halogenated organic compounds show excellent thermal conductivity, insulation, heat resistance, lipophilicity, and biological activity (Kim et al., 2020; Zakary et al., 2021). They are widely used in industrial, agricultural, medical, and military fields as cleaning agents, biocides, gasoline additives, solvents, degreasers, pesticides, and intermediates for chemical synthesis, yielding enormous economic and social benefits (Kurumbang et al., 2014; Zhang et al., 2019; Gul et al., 2020b; Ameen et al., 2021). However, increasing amounts of halogenated compounds are discharged into the environment due to overproduction and extensive use, which results in environmental contamination. These compounds spread in lakes, drinking water, groundwater, seawater, and soil. Unlike naturally occurring halogenated compounds, which can be used as antibiotics to treat bacterial infections, man-made halogenated compounds, which are used as degreasers, solvents, biocides, pharmaceuticals, cleaning agents, and in many other industrial applications, are dangerous when introduced to the environment (Wu et al., 2019; Kirkinci et al., 2021). This is because these compounds do not degrade easily in natural environments because of their chemical stability, resulting in their environmental accumulation. Moreover, these compounds can become concentrated and accumulate in organisms through the food chain, with carcinogenic, teratogenic, and mutagenic effects (Fan et al., 2020; Lou Y. Y. et al., 2021; Zhang C. et al., 2021). This poses a serious threat to human health and has become an issue of concern all over the world (Artabe et al., 2020; Lou Y. Y. et al., 2021). 
As the main decomposers in nature, microorganisms convert complex organic compounds into simple compounds, thus maintaining the cycle of elements that are vital to life (Hellal et al., 2021; Kajla et al., 2021; Yu et al., 2021). Microorganisms growing in environments polluted by organic halogenated compounds have the potential to transform these compounds owing to the presence of enzymes that catalyze dehalogenation in their cells, called dehalogenases (Atashgahi et al., 2018; Oyewusi et al., 2020b, 2021b). Among them, 2-haloacid dehalogenases are a family of critical enzymes that hydrolytically catalyze the dehalogenation of 2-haloacids to form corresponding 2hydroxy acids (Kurihara and Esaki, 2008; Adamu et al., 2020). They cannot only degrade toxic pollutants with low energy consumption but also have a wide substrate profile and high catalytic efficiency. They have highly chiral resolution properties, which may enable the production of optically pure 2halogenated and 2-hydroxyl compounds (Oyewusi et al., 2020a). Hence, 2-haloacid dehalogenases are highly valuable in the field of environmental remediation and environmentally friendly manufacturing of chiral chemicals. Here, we review the isolation source, classification, molecular structure, catalytic mechanism, catalytic properties, and industrial applications of 2-haloacid dehalogenases. These will enrich the biocatalytic repertoire of haloacid dehalogenases and broaden their applications and developments in the future.

\section{ISOLATION SOURCES AND CLASSIFICATION OF 2-HALOACID DEHALOGENASES}

Microorganisms possessing 2-haloacid dehalogenase are widespread in nature, and have been explored since the beginning of the 20th century. So far, increasing numbers of bacterial and fungal species capable of degrading halogenated xenobiotic pollutants have been isolated (Table 1). Most of these microorganisms were isolated from terrestrial environments, with only a few from marine environments, including Burkholderia sp. I37C (Chiba et al., 2009), Rhodobacteraceae sp. (Novak et al., 2013a), Psychromonas ingrahamii (Novak et al., 2013b), Pseudomonas stutzeri DEH130 (Zhang et al., 2013), Paracoccus sp. DEH99 (Zhang et al., 2014), Lysinibacillus boronitolerans MH2 (Heidarrezaei et al., 2020), and Bacillus megaterium BHS1 (Wahhab et al., 2020). The marine environment is the primary and optimal sink for halogenated pollutants because of their natural release by marine macroalgae, bacteria, sponges, tunicates, corals, worms, phytoplankton, and other invertebrates (Bidleman et al., 2019). Additionally, marine environments are considered extreme owing to a combination of unique properties including high pressure, high salinity, low temperature, oligotrophy, and special lighting conditions (de Oliveira et al., 2020; Ameen et al., 2021; Zhang J. et al., 2021). Because of this, microorganisms living in this environment are diverse and specific in gene composition and ecological functions; the intracellular enzymes of these microorganisms are correspondingly diverse and specific, conferring physiological and biochemical characteristics
TABLE 1 | The reported microorganisms degrading 2-haloalkanoic acids.

\begin{tabular}{|c|c|c|}
\hline Microorganisms & Genus & References \\
\hline \multirow[t]{23}{*}{ Bacteria } & Agrobacterium & Köhler et al., 1998 \\
\hline & Alcaligenes & Hill et al., 1999 \\
\hline & Ancylobacter & Kumar et al., 2016 \\
\hline & Arthrobacter & Bagherbaigi et al., 2013 \\
\hline & Azotobacter & Diez et al., 1996 \\
\hline & Bacillus & $\begin{array}{l}\text { Horisaki et al., 2011; } \\
\text { Ratnaningsih and Idris, 2018; } \\
\text { Oyewusi et al., 2021a }\end{array}$ \\
\hline & Burkholderia & Edbeib et al., 2020 \\
\hline & Klebsiella & Idris Ratnaningsih, 2015 \\
\hline & Lysinibacillus & Heidarrezaei et al., 2020 \\
\hline & Methylobacterium & Kurihara and Esaki, 2008 \\
\hline & Mesorhizobium & Zakary et al., 2021 \\
\hline & Moraxella & Kurihara et al., 2000 \\
\hline & Paracoccidioides & Satpathy et al., 2015 \\
\hline & Paracoccus & Zhang et al., 2014 \\
\hline & Pseudoalteromonas & Liao et al., 2015 \\
\hline & Pseudomonas & $\begin{array}{l}\text { Hasan et al., 1994; Park et al., } \\
\text { 2003; Schmidberger et al., 2008; } \\
\text { Zhang et al., } 2013\end{array}$ \\
\hline & Psychromonas & Novak et al., 2013b \\
\hline & Pyrococcus & Arai et al., 2006 \\
\hline & Rhizobium & $\begin{array}{l}\text { Adamu et al., 2016; Oyewusi } \\
\text { et al., 2020b }\end{array}$ \\
\hline & Rhodobacteraceae & Novak et al., 2013a \\
\hline & Serratia & Rosland Abel et al., 2012 \\
\hline & Sulfolobus & Xu et al., 2004 \\
\hline & Xanthobacter & van der Ploeg et al., 1991 \\
\hline \multirow[t]{9}{*}{ Fungi } & Beauveria & Satpathy et al., 2016 \\
\hline & Botrytis & Bustillo et al., 2003 \\
\hline & Candida & Polnisch et al., 1991 \\
\hline & Dichomitus & Muzikár et al., 2011 \\
\hline & Fusarium & Li et al., 2011 \\
\hline & Metarhizium & Satpathy et al., 2016 \\
\hline & Phanerochaete & Wang et al., 2009 \\
\hline & Pycnoporus & Muzikár et al., 2011 \\
\hline & Trichoderma & Bagherbaigi et al., 2013 \\
\hline
\end{tabular}

such as barophilicity, salt tolerance, cold adaptability, hyperthermostability, chemoselectivity, stereoselectivity, and regioselectivity (Thippeswamy et al., 2021; Zhang J. et al., 2021). The marine environment is therefore expected to be an important source of novel enzymes.

The 2-haloacid dehalogenases have been classified according to amino acid sequence conservation and substrate selectivity (Wang et al., 2018; Adamu et al., 2020). These enzymes are classified into four types according to their substrate specificities and product configurations: D-2-haloacid dehalogenase (DDEX, EC 3.8.1.9), L-2-haloacid dehalogenase (L-DEX, EC 3.8.1.2), configuration-inverting DL-2-haloacid dehalogenase (DL-DEXi, EC 3.8.1.10), and configuration-retaining DL-2haloacid dehalogenase (DL-DEXr, EC 3.8.1.11) (Zakary et al., 2021). D-DEX catalyzes the dehalogenation of D-2-haloalkanoic 
acids, whereas L-DEX specifically acts on L-2-haloalkanoic acids. DL-DEXi and DL-DEXr act on both enantiomers of substrates, but yield different product configurations. The 2haloacid dehalogenases in general are divided into Group I and Group II enzymes according to the amino acid sequence homology; D-DEX and DL-DEX belong to Group I and L-DEX to Group II.

\section{STRUCTURAL AND CATALYTIC CHARACTERISTICS OF 2-HALOACID DEHALOGENASES}

The structural diversity of 2-haloacid dehalogenases determines their diversity of function. The different types of 2-haloacid dehalogenases have different structures and catalytic mechanisms; an overview of this is provided in this section.

\section{L-DEX}

\section{Structural Characteristics and Catalytic Mechanism}

L-DEX specifically acts on L-2-haloalkanoic acids to produce D-2-hydroxyalkanoic acids. These enzymes are widespread in nature and their biochemical characteristics and structures have been studied extensively (Satpathy et al., 2016; Wang et al., 2016; Adamu et al., 2020). So far, the three-dimensional (3D) structures of specific L-DEXs and their substrate complexes have been analyzed, including L-DEX YL from Pseudomonas sp. strain YL (Hisano et al., 1996), DhlB from Xanthobacter autotrophicus GJ10 (Ridder et al., 1997), PH0459 from Pyrococcus horikoshii OT3 (Arai et al., 2006), DehIVa from Burkholderia cepacia MBA4 (Schmidberger et al., 2007), DehSft from Sulfolobus tokodaii (Rye et al., 2009) and DehRhb from Rhodobacteraceae (Novak et al., 2013a).

L-DEX is an $\alpha / \beta$ type hydrolase consisting of a typical Rossman-fold-like core domain and subdomain, with the active site located between the two domains (Figure 1), apart from $\mathrm{DhlB}$, which is composed of a core domain and two subdomains. Most L-DEX molecules are dimers consisting of two identical subunits, except for PH0459, which is a monomer according to its crystal structure (Arai et al., 2006). In a typical L-DEX structure, six-stranded parallel $\beta$-sheets (in order: $\beta 5-\beta 4-\beta 1-\beta 6-\beta 7-\beta 8$ ) are flanked on both sides by five $\alpha$-helices, forming three layers of $\alpha / \beta$ fold units together constituting a sandwich domain (Hisano et al., 1996; Poelarends and Whitman, 2010; Zhang et al., 2018). Although the core domain of L-DEX has an $\alpha / \beta$-type structure, it does not belong to the $\alpha / \beta$ hydrolase fold family, in which the typical domains are eight-stranded $\beta$-strands (in order: $\beta 1-\beta 2$ $\beta 4-\beta 3-\beta 5-\beta 6-\beta 7-\beta 8)$ with the $\beta 2$-strand antiparallel to the others. Two $\beta$-strands are separated by $\alpha$-helix from the third strand, forming a $\beta / \alpha / \beta$ unit. The first $\alpha$-helix and the last $\alpha$-helix are located at one side of the $\beta$-sheet, and the remaining $\alpha$-helices are at the other side (Janssen, 2004; Kunka et al., 2018; Babkova et al., 2020; Mazur et al., 2021).

The dehalogenation is catalyzed by L-DEX in an $\mathrm{S}_{\mathrm{N}} 2$ nucleophilic substitution reaction as confirmed by X-ray Crystallography, $\mathrm{O}^{18}$ isotope labeling, liquid chromatography-mass spectrometry (LC-MS), site-directed mutagenesis, and quantum mechanic/molecular mechanic (QM/MM) calculations (Adamu et al., 2017a,b). The carboxylic acid group of the aspartic acid residue acts as the nucleophile in the active center, which attacks the $\mathrm{C} 2$ atom of the L-2haloalkanoic acid to form an ester intermediate (Schmidberger et al., 2007). This intermediate product is then hydrolyzed by a water molecule, activated by His/Glu (in DehRhb) or Asn/Ser (in DehIVa) or Lys (in L-DEX YL) (Figure 2). The resultant halide ions are stabilized with the assistance of Arg or Asn or Phe. Greater numbers of halide ion acceptors can cleave stronger C-X bonds (Kurihara, 2011; Kondo et al., 2014).

\section{Biochemical Properties}

L-DEXs have been isolated from both terrestrial and marine environments. Some biochemical characteristics are shared between enzymes, and some differ. For example, L-DEX exhibits high catalytic activity on chlorinated and brominated substrates, but no such activity on D-2-haloalkanoic acids. Additionally, this enzyme cannot catalyze the dehalogenation of fluorinated and $\mathrm{C}_{3}$-substituted haloalkanoic acids. With the exception of L-DEX YL, L-DEXs only show high catalytic activity on haloalkanoic acids of two or three carbons in length, with low or no activity on haloalkanoic acids four or more carbons in length (van der Ploeg et al., 1991; Liu et al., 1994; Zhang et al., 2013, 2014).

L-DEX enzymes differ in substrate specificity; L-DEX YL is more specific to L-2-chloropropionic acid than chloroacetic acid, whereas the L-DEX from Bacillus strain I37C is more specific to chloroacetic acid than to 2-chloropropionic acid (Liu et al., 1994; Chiba et al., 2009). The optimal $\mathrm{pH}$ range for L-DEX reactions is 9-11 (alkaline). Subunit molecular weights range from 25 to 28 $\mathrm{kDa}$. Natural L-DEXs exist as monomers, dimers, and tetramers (van der Ploeg et al., 1991; Liu et al., 1994; Zhang et al., 2013, 2014).

L-DEXs isolated from different bacterial species have different thermal stability: the optimum reaction temperature for L-DEX from the terrestrial Pseudomonas putida is $30^{\circ} \mathrm{C}-45^{\circ} \mathrm{C}$, and it loses $50 \%$ activity after $15 \mathrm{~min}$ incubation at $55^{\circ} \mathrm{C}$. Psychromonas. ingrahamii is isolated from the sea-ice interface $\left(-10^{\circ} \mathrm{C}\right)$ and exhibits psychrophilic properties; the lowest temperature at which this strain is able to grow is $-12^{\circ} \mathrm{C}$. L-DEX Pin, from $P$. ingrahamii, has an optimum reaction temperature of $45^{\circ} \mathrm{C}$, with a melting temperature of $85^{\circ} \mathrm{C}$. L-DEX Pin possesses the characteristics of both psychrophilic and thermophilic enzymes. Structurally, compared with mesophilic enzymes, LDEX Pin has more hydrophobic surfaces and more salt bridges (Novak et al., 2013b).

The optimum reaction temperature for DehRhb, isolated from marine Rhodobacteraceae, is $55^{\circ} \mathrm{C}$. The activity of this enzyme remains at $\sim 45 \%$ after incubation for $1 \mathrm{~h}$ at $60^{\circ} \mathrm{C}$, indicating moderate thermal stability. Its key catalytic residues are His183 and Glu21, which are different from L-DEXs from terrestrial environments, suggesting that it may catalyze the dehalogenation with a novel catalytic mechanism (Novak and Littlechild, 2013). In summary, natural dehalogenases with novel properties may be more likely to be isolated from marine and other extreme environments; a greater understanding of their structures, catalytic mechanism and catalytic properties may 

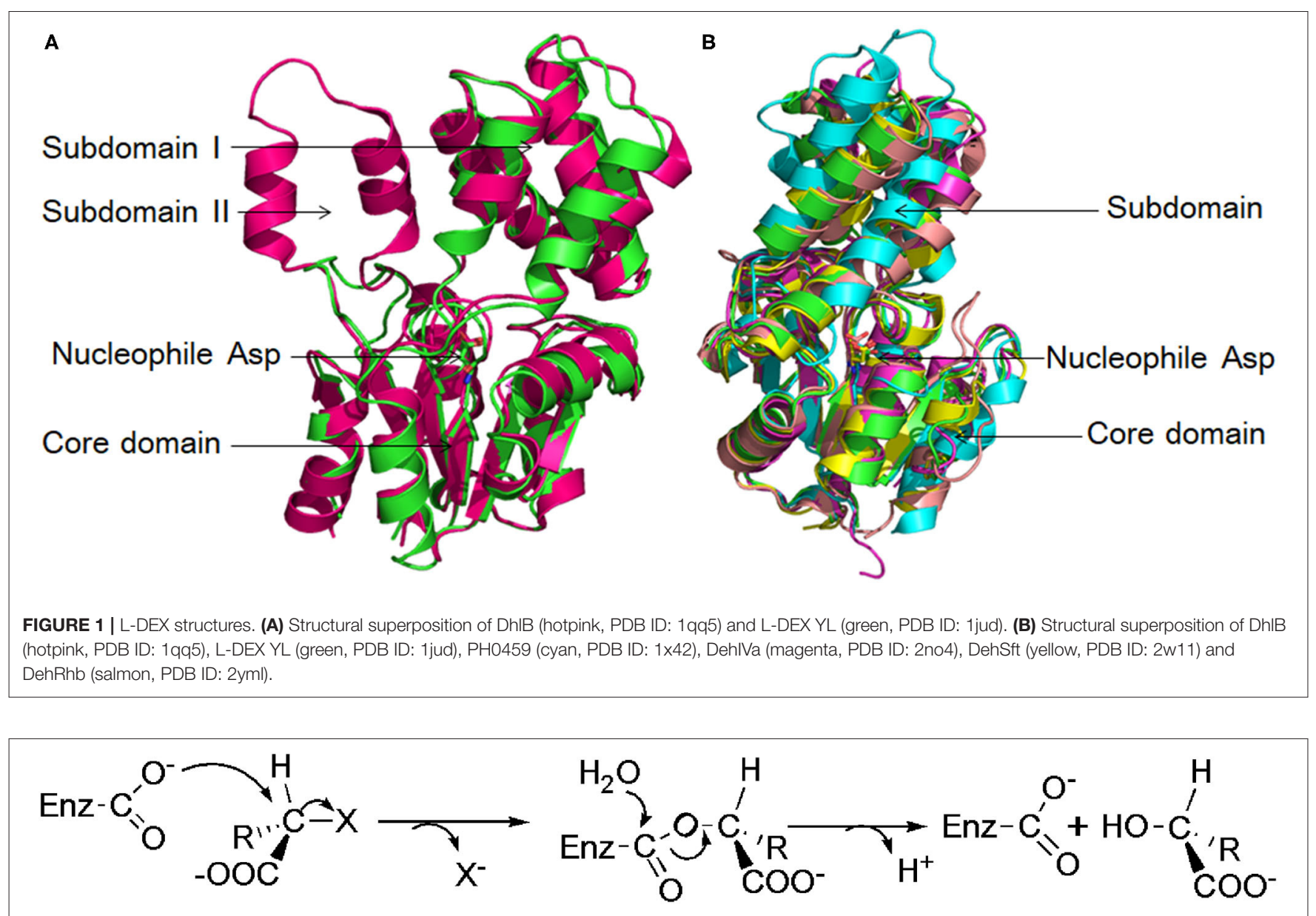

FIGURE 2 | Reaction mechanism of L-DEX (Schmidberger et al., 2007).

provide theoretical guidance for determining the direct evolution of L-DEXs and other dehalogenases.

\section{DL-DEX}

\section{Structural Characteristics and Catalytic Mechanism}

DL-DEX enzymes, which include DL-DEXi and DLDEXr, catalyze the hydrolytic dehalogenation of both enantiomers of 2-haloalkanoic acids to produce corresponding 2-hydroxyalkanoic acids.

For DL-DEXi, the configuration of the product is opposite to the substrate: the $\mathrm{C} 2$ atom of the substrate configuration is inverted during dehalogenation catalyzed by DL-DEXi. Six DLDEXi enzymes have been reported so far, including DL-DEX YL from Pseudomonas putida YL (Hasan et al., 1994; Soda et al., 1996), DL-DEX 113 from Pseudomonas sp. 113 (Nardi-Dei et al., 1999; Park et al., 2003), DehI from Pseudomonas putida PP3 (Park et al., 2003; Schmidberger et al., 2008), DL-DEX Mb from Methylobacterium sp. CPA1 (Siwek et al., 2013), DehE from Rhizobium sp. RC1 (Hamid et al., 2011; Zainal Abidin et al., 2019), and DhIIV from Alcaligenes xylosoxidans ABIV (Brokamp et al., 1996; Hamid et al., 2011). The crystal structures of DehI and DL-DEX Mb have been studied, revealing that DL-DEXi is an $\alpha$-helical hydrolase, with no structural homology to L-DEX and other fold superfamilies in the hydrolases (Schmidberger et al., 2008; Siwek et al., 2013).

As shown in Figure 3, DehI is a homodimer according to its crystallographic structure. The $\mathrm{N}$-terminus (amino acid residues 1-130) and C-terminus (residues 166-296) share 16\% sequence identity in monomers, which form a pseudo-dimer. The active site is located at the interface of the pseudo-dimer, which binds D- and L- substrates (Schmidberger et al., 2008). The catalytic mechanism of DL-DEXi is different to that of L-DEX: dehalogenation catalyzed by D-DEXi is directly mediated by an activated water molecule, without involving the formation of E-S ester intermediate (Figure 4) (Nardi-Dei et al., 1999). The nucleophilic water molecule is likely activated by the conserved Asp and Asn residues; however, there is no relevant experimental evidence for this.

The transformation of the $C_{2}$-configuration of the substrate catalyzed by DL-DEXr is opposite to that of DL-DEXi. DL-DEXr catalyzes dehalogenation with retention of the $C_{2}$-configuration of the substrate. Therefore, the substrate and product share the same configuration. DL-DEXr has so far only been reported in P. putida PP3 (Weightman et al., 1982; Park et al., 2003). 


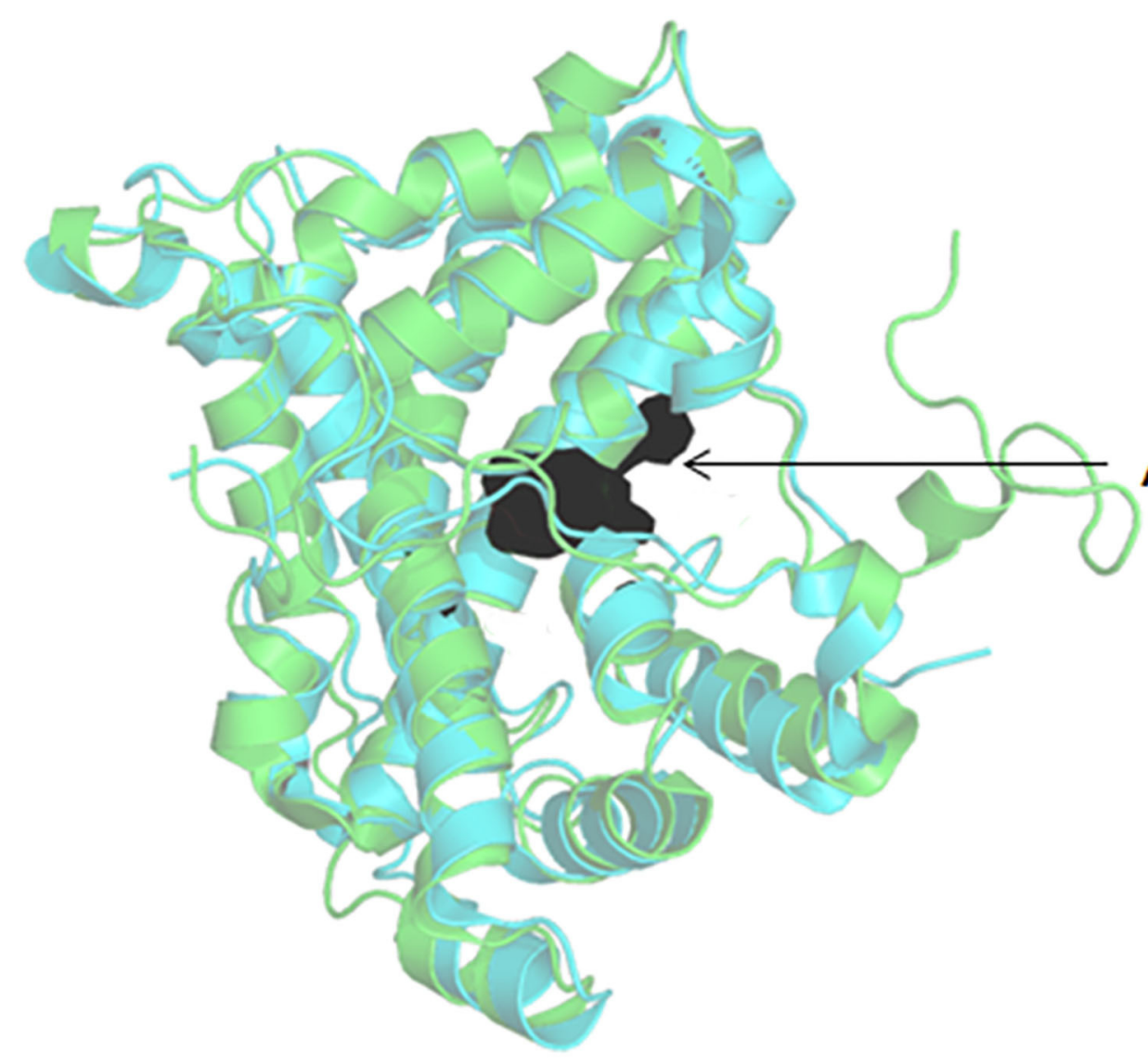

Active pocket

FIGURE 3 | Structural superposition of Dehl (green, PDB ID: 3bjx) and DL-DEX Mb (cyan, PDB ID: 4n2x). The active pocket is shown as surface.

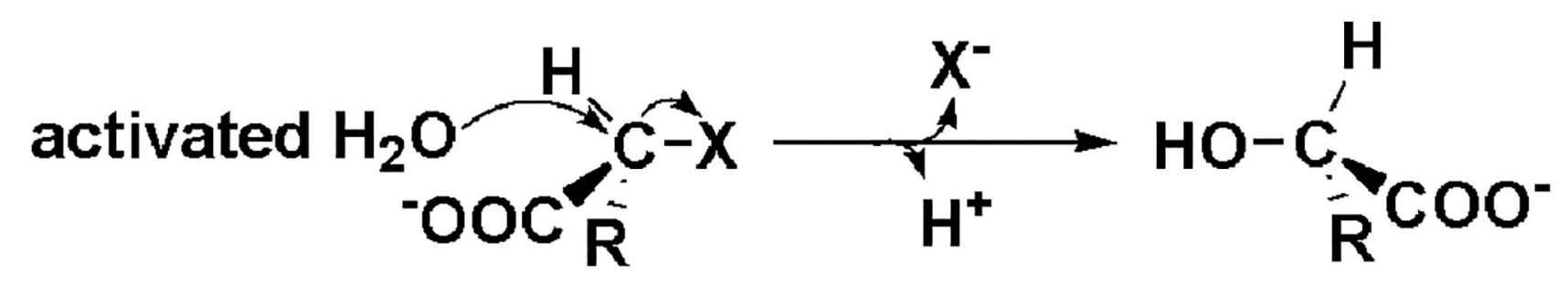

FIGURE 4 | Reaction mechanism of DL-DEXi (Nardi-Dei et al., 1999).

Gene sequence information for this enzyme is still unknown, and the reaction mechanism has not been analyzed. It is proposed that dehalogenation involves a cysteine residue, as DL-DEXr is highly sensitive to sulfhydryl reagents such as $N$-ethylmaleimide and $p$-chloromercuribenzoic acid. The reaction is thought to proceed with double inversion of the $C_{2}$-configuration of the substrate, resulting in the retention of the $C_{2}$-configuration: the first $C_{2}$-configuration inversion releases halogen ions and forms an E-S thioester intermediate; then, the intermediate is hydrolyzed under the attack of a water molecule, and the $C_{2}$ configuration is reversed again. However, there is currently no direct experimental data to confirm this hypothesis (Figure 5) (Weightman et al., 1982).

\section{Biochemical Properties}

In DL-DEXs, only DehI, DehE, DL-DEX 113 and DL-DEX ABIV have been characterized in terms of their enzymatic properties (Brokamp et al., 1996; Schmidberger et al., 2008). These enzymes have a greater specificity for L-2-haloalkanoic acids than D-2-haloalkanoic acids (Table 2). DL-DEXi can catalyze the dehalogenation of haloalkanoic acids with a carbon chain length of two to four, and catalyzes the formation of oxalate from trichloroacetate (Soda et al., 1996). Most DL-DEXi enzymes are homodimers, except for DL-DEX YL, which is a monomer. The subunit molecular weight ranges from 26 to 36 $\mathrm{kDa}$ (Kondo et al., 2014). DL-DEXi maximum activity levels occur at a $\mathrm{pH}$ of $\sim 9.5$. The optimum reaction temperature is 


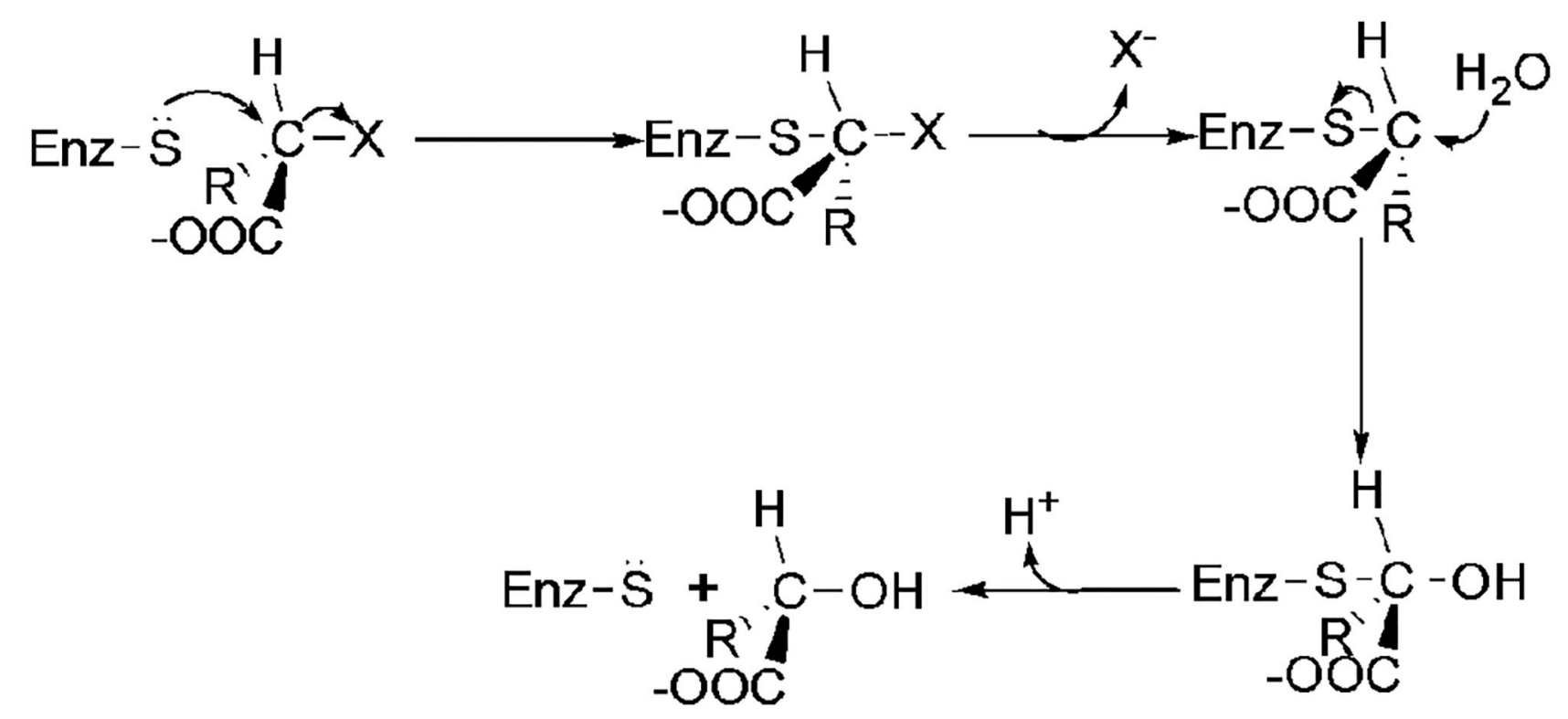

FIGURE 5 | Possible mechanism of DL-DEXr involving the retention of $C_{2}$-configuration of the substrate (Weightman et al., 1982 ).

TABLE 2 | Enatioselectivity of DL-DEXis from different strains.

\begin{tabular}{lccl}
\hline Enzymes & Strains & L/D $^{\text {a }}$ & References \\
\hline Dehl & P. putida PP3 & 1.2 & Park et al., 2003 \\
DehE & R. sp. RC1 & 1.6 & Hamid et al., 2011 \\
DL-DEX 113 & P. sp. 113 & 1.4 & Park et al., 2003 \\
DhIIV & A. xylosoxidans ABIV & 1.1 & Brokamp et al., 1996 \\
DL-DEX YL & P. putida YL & - & \\
DL-DEX Mb & M. sp. CPA1 & - & \\
\hline
\end{tabular}

a $L / D$, the ratio of catalytic activity on L-2-chloropropionic acid and D-2-chloropropionic acid; -, no experimental data is available.

between 30 and $40^{\circ} \mathrm{C}$ (Leigh et al., 1986; Park et al., 2003; Hamid et al., 2011). DL-DEXr is sensitive to SH-reagents; like DLDEXi, it degrades haloalkanoic acids with a chain length of 2-4 (Weightman et al., 1982).

\section{D-DEX}

\section{Structural Characteristics and Catalytic Mechanism}

D-DEXs specifically catalyze the hydrolytic dehalogenation of D2-haloalkanoic acids to produce L-2-hydroxyacids. So far, only four kinds of primary structure information are available for D-DEX, including DehD from Rhizobium sp. RC1 (Sudi et al., 2014), DehII from Agrobacterium sp. NHG3 (Higgins et al., 2005), HadD AJ1 from Pseudomonas putida AJ1 (Smith et al., 1990) and DehDIV-R from Pseudomonas sp. ZJU26 (Wang Y. et al., 2020). HadD AJ1 and DehDIV-R share the highest sequence homology (89\%); HadD AJ1 and DehII NHG3 share 22.2\% sequence homology, and HadD AJ1 and DehD share $32.6 \%$ sequence homology.
The author has extensively studied on the structure and catalytic mechanism of HadD AJ1. The crystal structure of HadD AJ1 is highly similar to that of DL-DEXi. Both types of enzymes are $\alpha$-helical proteins, different from the $\alpha / \beta$ fold structure. HadD $\mathrm{AJ} 1$ is a homotetramer according to its crystallographic structure; each monomer comprises two repeats with $20 \%$ sequence identity (Figure 6). The two repeated folds are composed of N-terminal $\alpha$-helices 1-6 and C-terminal $\alpha$-helices $7-12$, respectively, with a linker section containing 33 amino acids and a 310 -helix $\eta_{1}$ (Figure 6A). These two repeats are stabilized by van der Waals forces, salt bonds, hydrogen bonds, and hydrophobic interactions. As shown in Figure 6B, helix $\alpha_{4}$ and $\alpha_{10}$ are arranged in parallel with each other, and $\alpha_{6}$ and $\alpha_{12}$ cross each other at the bulge between them. Helices $\alpha_{6}$ and $\alpha_{12}$ mutually interlace at their bulges, located in the middle of the helices (Wang et al., 2018). This has been reported in many proteins with internal structural repeats, which are considered to result from genetic processes such as fusion and fission of domains and gene duplication during protein evolution (Longo et al., 2014; Berezovsky et al., 2017; Vrancken et al., 2020).

In HadD AJ1, Asp205 is the key catalytic residue, activating the water molecule with the assistance of Asn131. This was identified through an analysis of the complex structure of wildtype (WT) enzyme binding the product L-lactic acid (L-LA) and a D205N mutant binding the substrate D-2-chloropropionate (D-2-CPA) (Figure 7A). The dehalogenation catalyzed by D-DEX is directly mediated by activated water molecules, without forming an ester intermediate in the reaction process; this is the same process as DL-DEXi (Figure 7B). The activated water molecule attacks the $\mathrm{C} 2$ atom of the substrate from the opposite side of the halogen atom, breaking the $\mathrm{C}-\mathrm{X}$ bond (Figure 7A). The halogen ion is released toward F281, and simultaneously, the hydroxyl group of the activated water molecule is bonded 


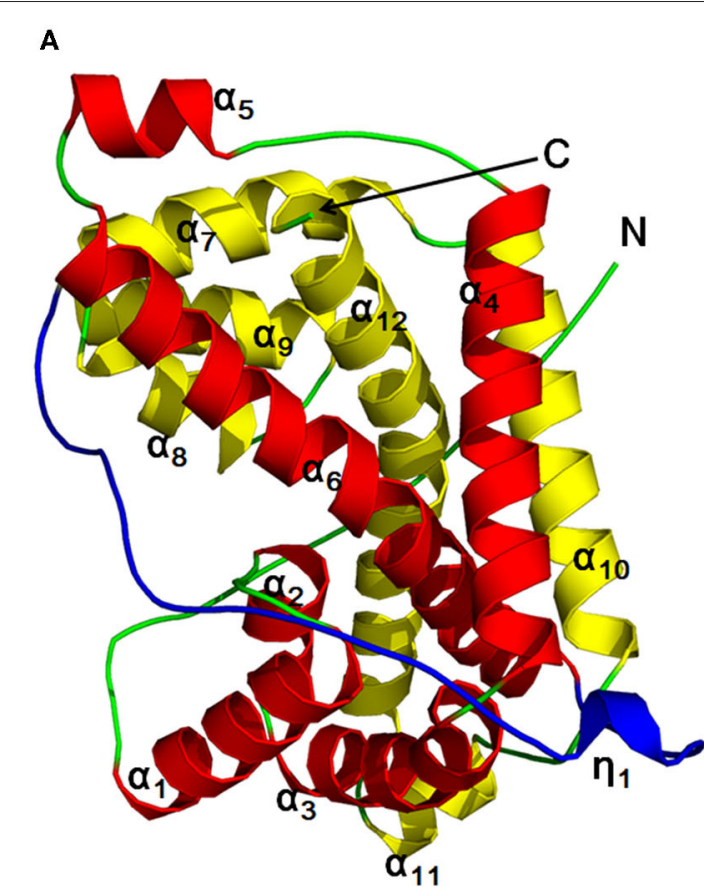

B

FIGURE 6 | HadD AJ1 monomer (Wang et al., 2018). (A) Ribbon representation of monomeric HadD AJ1 composed of two repeats (repeat 1: red; repeat 2: yellow) and a linker (cyan). (B) 3D superposition of repeat 1 (red) and repeat 2 (yellow).

to the $\mathrm{C} 2$ atom of the substrate to form L-lactic acid (Wang et al., 2018).

D-DEX and DL-DEXi share high amino acid sequence as well as structural homology. Moreover, both types of enzymes catalyze dehalogenation by the same mechanism, directly mediated by the nucleophilic water molecule; this differs from dehalogenation catalyzed by L-DEX, which is mediated by E$S$ ester intermediates. This suggests an evolutionarily close relationship between D-DEX and DL-DEXi.

\section{Biochemical Properties}

Currently, there are only a few studies on D-DEX enzymes, likely a result of the lack of microorganisms known to produce D-DEX. From analyses of DehD and HadD AJ1 biochemical properties, D-DEXs specifically catalyze dehalogenation of D-2-chlorinated and D-2-brominated acids with carbon chain lengths of 2-4. However, D-DEX has a higher catalytic activity on brominated than chlorinated substrates (Smith et al., 1990; Huyop and Sudi, 2012). $K_{\mathrm{m}}$ values of DehD, HadD AJ1, and DehDIV-R are 0.06, 0.94 , and $2.2 \mathrm{mmol} / \mathrm{L}$, respectively, with D-2-CPA as the substrate (Smith et al., 1990; Huyop and Sudi, 2012; Wang Y. et al., 2020). Compared with HadD AJ1 and DehDIV-R, DehD has a stronger affinity for D-2-CPA.

The natural active states of D-DEXs are different: DehD exists is a homodimer, while HadD AJ1 is a homotetramer. The optimal reaction $\mathrm{pH}$ of D-DEXs ranges from 9.0 to 10.0. The enzyme activity decreases rapidly when the $\mathrm{pH}$ falls outside the range of 8.0-10.0; under these conditions, HadD AJ1 exhibits $<50 \%$ catalytic activity (Smith et al., 1990). In comparison with L-DEXs,
D-DEXs are mesophilic, with an optimal reaction temperature of $50^{\circ} \mathrm{C}-60^{\circ} \mathrm{C}$; however, the enzyme molecules are relatively stable between 30 and $40^{\circ} \mathrm{C}$, but rapidly lose activity in a reaction temperature higher than $40^{\circ} \mathrm{C}$ (Smith et al., 1990).

\section{APPLICATION}

The 2-haloacid dehalogenases can detoxify halogenated pollutants by hydrolysis without the addition of other reductive agents; for this reason, their potential application in bioremediation is particularly attractive (Behbahani et al., 2018; Oyewusi et al., 2020b, 2021b; Zakary et al., 2021). The 2-haloacid dehalogenases are also highly stereoselective, and they may therefore be valuable in fine chemistry synthesis applications (Chen and Ribeiro de Souza, 2019; Adamu et al., 2020; Wang S. et al., 2020). These enzymes can be used to obtain chiral hydroxy acids and haloalkanoic acids with low molecular weights; these small organic acids generally act as intermediates for synthesizing agrochemicals, medicines, and other important chemicals (Leemans Martin et al., 2020; Gurushankara, 2021). Hence, 2-haloacid dehalogenases are promising and potentially highly valuable for their application in environmental remediation and chemical synthesis (Bommarius, 2015; Tanokura et al., 2015; Zhang et al., 2018); here, we discuss the main fields in which they could be applied.

\section{Environmental Bioremediation}

Halogenated carboxylic acids such as 2-chloropropionic acids and 2,2-chloropropionic acids are widely used as an 
A
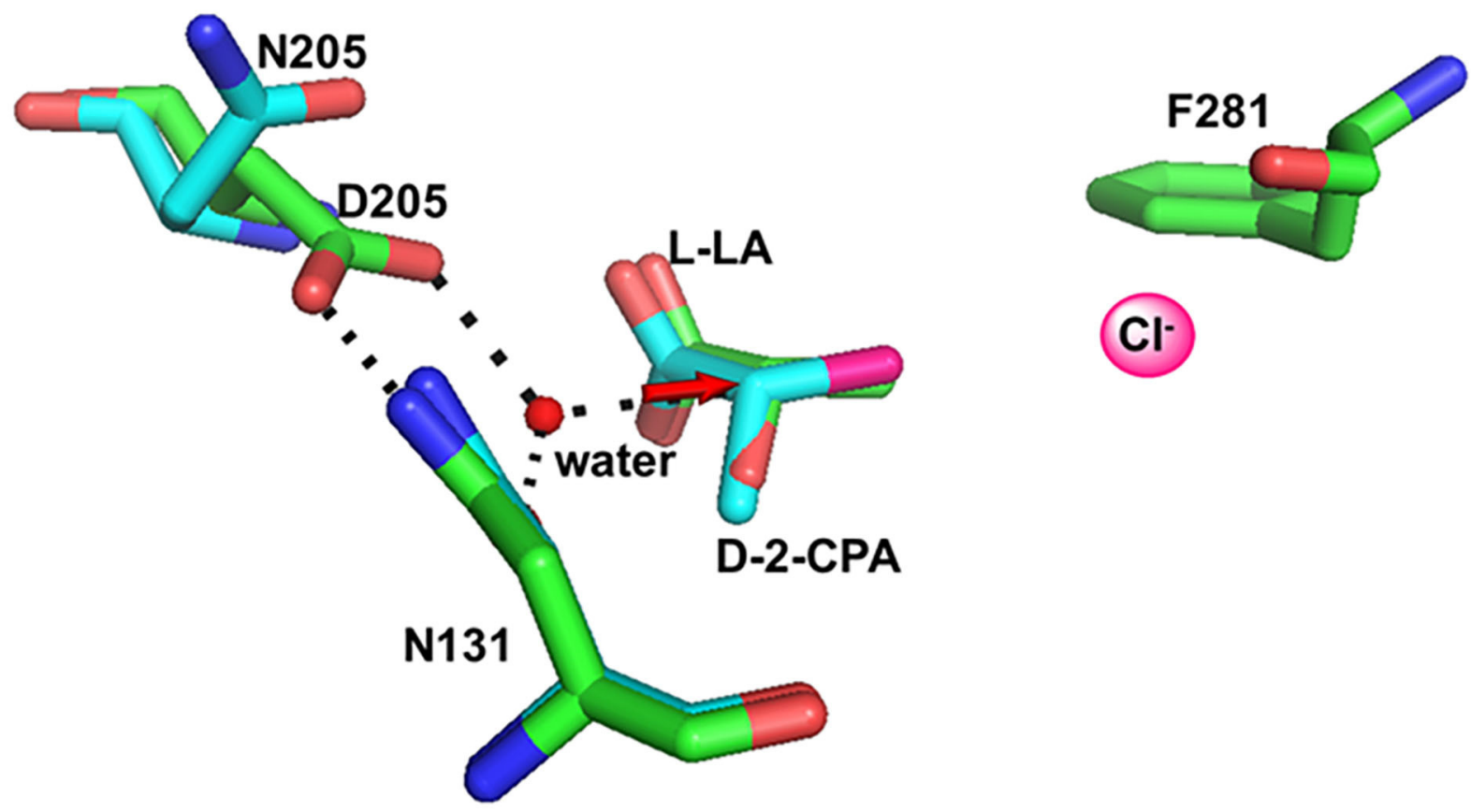

CF

B
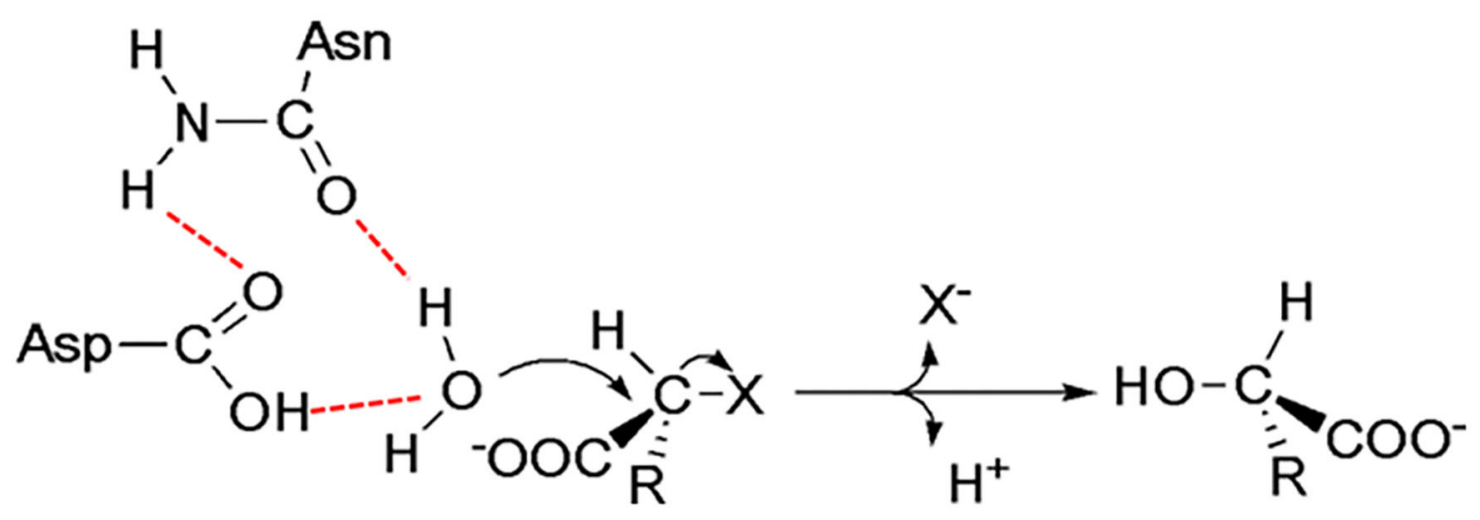

FIGURE 7 | The molecular mechanism of dehalogenation catalyzed by D-DEX. (A) Structural superimposition of WT/L-LA (green, PDB ID: 5gzy) and D205N/D-2-CPA (cyan, PDB ID: 5gzx) complex. (B) Reaction mechanism of D-DEX (Wang et al., 2018).

intermediate in the synthesis of pesticides and pharmaceuticals, especially the chirally pure 2-chloropropionic acid precursors for synthesizing many chiral drugs (Nguyen et al., 2021; Zhou et al., 2021). However, these haloacids produce chlorinated organic contaminants owing to extensive use and improper disposal. Haloacids are also intermediates in the degradation of some halogenated compounds, such as 1,2-dichloroalkane and hexachlorocyclohexane, which results in more haloacid contaminants in the environment (Hermon et al., 2018). The accumulation of these pollutants causes serious environmental problems and threats to human and other organisms' health. The 2-haloacid dehalogenase can catalyze the dehalogenation of 2-chloropropionic acids and 2,2-dichloropropionic acids to form non-toxic hydroxyl acids, which is a very promising potential tool for environmental bioremediation (Oyewusi et al., 2021b; Zakary et al., 2021). Dioxin compounds are carcinogenic byproducts originating from natural and anthropogenic sources such as herbicides, pesticides, and combustion processes; high levels of dioxin-contamination have been reported in food, soils, and blood samples of local residents in Southern Vietnam (Nguyen et al., 2021). Burkholderia cenocepacia strain 869T2 can degrade $0.2 \mathrm{mg} \mathrm{L}^{-1}$ of dioxin within 1 week under aerobic conditions, in which L-2-haloacid dehalogenase plays a crucial role (Nguyen et al., 2021). Haloacetic acids are the second most prominent class of disinfection by-products, and are frequently detected in surface and drinking water systems. These compounds have genotoxic, mutagenic, cytotoxic, and tumorigenic effects in humans (Kim et al., 2020; Long et al., 2021; Lou J. et al., 2021). In metabolically engineered Burkholderia species, the degradation activity of haloacetic acids can be increased by $4-8$ 
times (Su et al., 2013). The bacterial degradation of haloacetic acids was found to be affected by water distribution system conditions, including $\mathrm{pH}$, phosphate, total organic carbon and residual chlorine (Behbahani et al., 2018). The order of mean haloacetic acid degradation rates has been found to be $\mathrm{di}>$ mono $>$ tri-halogenated acetic acids.

Phytoremediation has been attracting more attention as an environmentally friendly technology to clean up environmental contamination (Kurade et al., 2021); transgenic tobacco that produces haloalkane dehalogenase and haloacid dehalogenase, and which therefore contains a complete degradation pathway, has been reported to degrade 1,2-dichloroethane (Mena-Benitez et al., 2008).

\section{Fine Chemical Synthesis}

The growing interest in the use of 2-haloacid dehalogenases in fine chemical synthesis is due to their chiral selectivity. Optically pure compounds are generally synthesized using chemical methods; however, this is unpopular owing to the involvement of toxic reagents, as well as the low yield and low optical purity of products (Santi et al., 2021). Biocatalysis is considered as a more environmentally friendly and effective method because of the mild reaction conditions, and remarkable enantioselectivity (Novak et al., 2013b; Schober and Faber, 2013; Wang S. et al., 2020).

L-2-chloropropionic acid is an important precursor in the synthesis of herbicides and pesticides (Zhou et al., 2021). DDEX specifically hydrolyzes D-2-chloropropionic acid in racemic 2-chloropropionic acid; L-2-chloropropane acid is therefore obtained with high enantiomeric purity by separation (Gong et al., 2018). To obtain optically active L-2-chloropropionic acid, Imperial Chemical Industries has already applied HadD AJ1 to the resolution of racemic 2-chloropropionic acid in an industrial setting, which has been the primary method for producing chiral chloropropionic acid (Taylor Stephen, 1985; Parker and Colby, 1995). It has also been used by AstraZeneca in the resolution of rac-2-CPA by D-DEX. This method is also suitable for the production of other short-chain chiral 2-halogenated acids, and the scale can be higher than 1,000 tons/year (Schober and Faber, 2013).

D-2-CPA is an important raw material for chemical synthesis that can be directly used to produce a variety of pharmaceutical intermediates, such as the nutritional medicine L-alanyl-L-glutamine and the anti-tuberculosis drug thiolactomycin. L-DEX can be used for the resolution of racemic 2-chloropropionic acids to obtain D-2-CPA with enantiomeric purity (Breuer et al., 2004).

Optically pure lactic acid is an important chiral intermediate in the synthesis of agrochemical, pharmaceutical, and chemical industries; it has been reported that L-lactic acid can be used to synthesize nanoparticles and nanofibers, which act as drug carriers (Chuan et al., 2020; Liu et al., 2021; Ma et al., 2021; Yavari Maroufi et al., 2021). D-lactic acid is also involved in the synthesis of important chiral drug intermediates, such as methyl D-lactate (Sengupta et al., 2020). Xie and colleagues studied the reaction conditions of L-DEX from thermophilic archaea Sulfolobus tokodaii in the catalytic conversion of racemic 2-chloropropionic acid to D-lactic acid (Rye et al., 2009; Xie et al., 2015); after optimizing reaction conditions with regard to substrate, buffer, and enzyme concentration, preparation of D-lactic acid was found to work best with $0.5 \mathrm{~mol} / \mathrm{L} 2$-chloropropionic acid.

D-2-bromobutyric acid is used as an intermediate for the synthesis of pharmaceuticals and agrochemicals. The fluoroacetate dehalogenase mutant H155V/W156R/Y219M is reported to catalyze the kinetic resolution of rac-2-bromobutyric acid, producing D-2-bromobutyric acid with an enantiomeric excess of $99.7 \%$ (Wang S. et al., 2020).

\section{Agricultural Production}

Herbicides with broad spectrum can effectively remove a variety of weeds, such as monochloroacetic acid, 2-chloropropionic acid, and 2-dichloropropionic acid. However, these herbicides can also damage economically valuable crops, resulting in significant losses in agricultural production. These losses can be avoided by developing herbicide-resistant crops, which requires the introduction of genes encoding dehalogenases into these crops. The dehD gene from Rhizobium sp. RCI, encoding D2-haloacid dehalogenase, has been successfully introduced into tobacco as selective tag, constructing a transgenic variant of Nicotiana benthamiana with anti-monochloroacetic acid activity (Mohamed et al., 2020). This transgenic, herbicide-resistant tobacco is confirmed to be effective at various development stages, including seed germination and mature leaf stages. The dehalogenase gene is therefore likely to play an important role as a dominant, selectable marker gene for the construction of other crop species resistant to broad-spectrum halogenated compound herbicides.

\section{Other Fields}

Dehalogenases can also be used to construct biosensors for in situ detection of organic halogenated pollutants in the environment (Artabe et al., 2020; Gul et al., 2020a,b). By immobilizing halohydrin dehalogenase on a glass fiber membrane, detection limits of $0.06 \mathrm{mmol} / \mathrm{L} \mathrm{1,3-dichloro-2-propanol} \mathrm{and} 0.09 \mathrm{mmol} / \mathrm{L}$ 2,3-dibromo-1-propanol have been achieved (Gul et al., 2020b). A detection limit of $1 \mathrm{mg} / \mathrm{L}$ dichlorethane has been achieved by immobilizing haloalkane dehalogenase on stacked chitosan films (Shahar et al., 2019a,b).

Dehalogenases act as tags when genetically fused to a protein of interest, termed HaloTag technology (England et al., 2015; Döbber and Pohl, 2017; Erdmann et al., 2019). This technology overcomes the current limitations of traditional protein tagging platforms, as it can be applied to protein isolation and purification, studies of protein synthesis and degradation, analyses of protein function, studies of protein-protein and protein-DNA interactions, and molecular and cellular imaging (Encell et al., 2012; Merrill et al., 2019; Cattoglio et al., 2020; Freitas et al., 2021; Minner-Meinen et al., 2021). Furthermore, novel technologies have been developed for tumor diagnosis and treatment involving the linkage of dehalogenase fused with cancer cell recognition peptides to multifunctional nanoparticles (Garbujo et al., 2020). 


\section{DISCUSSION AND PROSPECTS}

A variety of 2-haloacid dehalogenases have so far been isolated and identified. Although structural information and catalytic mechanisms for L-DEX, DL-DEXi, and D-DEX have generally been well-understood, very little information on the structure and catalytic mechanism of DL-DEXr is available. Therefore, further study is necessary to understand DL-DEXr.

Enzyme stereoselectivity has been attracting a great deal of attention for asymmetric synthesis and chiral resolution. The 2-haloacid dehalogenases show typical stereoselectivity; however, little is known about the stereoselective mechanism. The enantioselective mechanism of L-DEX has been studied using quantum mechanics/molecular mechanics (QM/MM) and fragment molecular orbital calculation (Kondo et al., 2015; Adamu et al., 2019), which have confirmed that the high activation energy barrier prevents this enzyme from acting on the $D$-substrate. However, it is still unclear how selectivity of enzymes on chiral substrates is regulated. The stereoselective mechanism of D-DEX has been studied, and enzymatic stereoselectivity was found to be controlled by the residue Leu288, which determines the entry of L-substrate into the active site of the enzyme with steric hindrance. The mutation of residue leucine to isoleucine enables the enzyme to catalyze the dehaologenation of the L-substrate, owing to the different rotation position of Ile288 compared with Leu288. In the mutant enzyme, Ile288 functions as a wing gatekeeper, interacting with the substrate by gate-flipping during dehalogenation, allowing the L-substrate to enter the active site. However, it is still unclear how DLDEXr and DL-DEXi recognize and interact with chiral substrates. Stereoselective properties make biocatalysts valuable in the preparation of optically pure compounds, which is an important area of environmentally friendly chemistry. An ideal industrial biocatalyst should have both high catalytic activity and specific stereoselectivity; exploring the molecular regulatory mechanisms underlying these properties forms the basis of artificial customization of dehalogenases with these properties. Reactions can be controlled using direct regulation of enzyme selectivity, forming products with high optical purity and unique structures. Further study on the stereoselectivity of 2-haloacid dehalogenase is therefore required in order to successfully manipulate this property.

Most 2-haloacid dehalogenases have a high catalytic activity with short-chain halogenated acid substrates containing fewer than four carbon atoms, while they show weak or no catalytic activity for longer-chain halogenated acids. Additionally, the low tolerance of these enzymes to organic solvents limits the range of their substrate profile. In order to obtain enantiomerically pure chiral products, enzymatic catalysis is sometimes used in enantiomeric resolution by combining with chemical convergence (Clayton et al., 2020). However, the conditions of the enzymatic reaction are incompatible with the high temperature and extreme $\mathrm{pH}$ required for chemical hydrolysis in the downstream separation process. Therefore, it remains necessary to identify novel 2-haloacid dehalogenases with unique properties, allowing them to function in these more extreme conditions (Marshall et al., 2021). Marine microorganisms may be the primary source of novel enzymes with extraordinary properties owing to their previously established genetic and biochemical diversity.

The birth of protein engineering technology has opened up a new route for researchers to develop excellent biocatalysts by redesigning natural enzymes (Marshall et al., 2021; Watanabe et al., 2021; Xiong et al., 2021). Many enzyme engineering design strategies have emerged, such as directed evolution, rational, semi-rational, de novo, computer-assisted, and artificial intelligence (Bunzel et al., 2021; Narayanan et al., 2021; Tunyasuvunakool et al., 2021; Woolfson, 2021; Wu et al., 2021). These strategies have been used to improve enzyme stability, activity, and selectivity for substrates. However, so far, only L-2-haloacid dehalogenases have been engineered to alter their substrate specificity. The mutation of residue Ser188 to Val in the enzyme DehE enables it to act on 3-chloropropionic acid (Hamid et al., 2015). Recent developments in understanding the structural and catalytic properties of 2-haloacid dehalogenases will also likely enable these enzymes to be more easily modified for commercial uses alongside L-2-haloacid dehalogenases. Given this overall direction of research, an increasing variety of 2-haloacid dehalogenases will likely be modified through protein engineering techniques to improve their properties for biotechnological applications.

\section{AUTHOR CONTRIBUTIONS}

YW and QX handled the literature collection and literature research. YW prepared the original draft. QZ, JX, and DP modified the manuscript. All authors critically reviewed, contributed to, and approved the final manuscript.

\section{FUNDING}

This work was supported by the National Natural Science Foundation of China (22078308), Innovation Leadership Program in Sciences and Technologies for Central Plains Talent Plan (214200510009), Key Scientific Research Projects in the Universities of Henan Province (20A180024), Research Project of Shangqiu Normal University (700144), Postdoctoral Science Foundation of Henan Province (50026003), Program for Science and Technology Innovative Research Team in University of Henan Province (21IRTSTHN025), and Innovation Leadership Program in Sciences and Technologies for Zhengzhou Talent Gathering Plan, Henan Academician Workstation for Industrial Technology of Dry Chilli.

\section{ACKNOWLEDGMENTS}

We would like to thank the reviewers for their constructive comments. 


\section{REFERENCES}

Adamu, A., Abdul Wahab, R., Aliyu, F., Abdul Razak, F. I., Mienda, B. S., Shamsir, M. S., et al. (2019). Theoretical analyses on enantiospecificity of L-2-haloacid dehalogenase (DehL) from Rhizobium sp. RC1 towards 2-chloropropionic acid. J. Mol. Graph Model. 92, 131-139. doi: 10.1016/j.jmgm.2019.07.012

Adamu, A., Shamsir, M. S., Wahab, R. A., Parvizpour, S., and Huyop, F. (2017a). Multi-template homology-based structural model of L-2-haloacid dehalogenase (DehL) from Rhizobium sp. RC1. J. Biomol. Struct. Dyn. 35, 3285-3296. doi: 10.1080/07391102.2016.1254115

Adamu, A., Wahab, R. A., Aliyu, F., Aminu, A. H., Hamza, M. M., and Huyop, F. (2020). Haloacid dehalogenases of Rhizobium sp. and related enzymes: Catalytic properties and mechanistic analysis. Process Biochem. 92, 437-446. doi: 10.1016/j.procbio.2020.02.002

Adamu, A., Wahab, R. A., and Huyop, F. (2016). L-2-Haloacid dehalogenase (DehL) from Rhizobium sp. RC1. SpringerPlus 5:695. doi: 10.1186/s40064-016-2328-9

Adamu, A., Wahab, R. A., Shamsir, M. S., Aliyu, F., and Huyop, F. (2017b). Deciphering the catalytic amino acid residues of L-2-haloacid dehalogenase (DehL) from Rhizobium sp. RC1: an in silico analysis. Comput. Biol. Chem. 70, 125-132. doi: 10.1016/j.compbiolchem.2017.08.007

Ameen, F., AlNadhari, S., and Al-Homaidan, A. A. (2021). Marine microorganisms as an untapped source of bioactive compounds. Saudi J. Biol. Sci. 28, 224-231. doi: 10.1016/j.sjbs.2020.09.052

Arai, R., Kukimoto-Niino, M., Kuroishi, C., Bessho, Y., Shirouzu, M., and Yokoyama, S. (2006). Crystal structure of the probable haloacid dehalogenase PH0459 from Pyrococcus horikoshii OT3. Protein Sci. 15, 373-377. doi: 10.1110/ps.051922406

Artabe, A. E., Cunha-Silva, H., and Barranco, A. (2020). Enzymatic assays for the assessment of toxic effects of halogenated organic contaminants in water and food. A review. Food Chem. Toxicol. 145, 111677-111697. doi: 10.1016/j.fct.2020.111677

Atashgahi, S., Liebensteiner, M. G., Janssen, D. B., Smidt, H., Stams, A. J. M., and Sipkema, D. (2018). Microbial synthesis and transformation of inorganic and organic chlorine compounds. Front. Microbiol. 9:3079. doi: 10.3389/fmicb.2018.03079

Babkova, P., Dunajova, Z., Chaloupkova, R., Damborsky, J., Bednar, D., and Marek, M. (2020). Structures of hyperstable ancestral haloalkane dehalogenases show restricted conformational dynamics. Comput. Struct. Biotechnol. J. 18, 1497-1508. doi: 10.1016/j.csbj.2020.06.021

Bagherbaigi, S., Gicana, R., Lamis, R. J., Nemati, M., and Huyop, F. (2013). Characterisation of Arthrobacter sp. S1 that can degrade $\alpha$ and $\beta$-haloalkanoic acids isolated from contaminated soil. Ann. Microbiol. 63, 1363-1369. doi: 10.1007/s13213-012-0595-4

Behbahani, M., Lin, B., Phares, T. L., and Seo, Y. (2018). Understanding the impact of water distribution system conditions on the biodegradation of haloacetic acids and expression of bacterial dehalogenase genes. J. Hazard. Mater. 351, 293-300. doi: 10.1016/j.jhazmat.2018.02.047

Berezovsky, I. N., Guarnera, E., and Zheng, Z. (2017). Basic units of protein structure, folding, and function. Prog. Biophys. Mol. Bio 128, 85-99. doi: 10.1016/j.pbiomolbio.2016.09.009

Bidleman, T. F., Andersson, A., Jantunen, L. M., Kucklick, J. R., Kylin, H., Letcher, R. J., et al. (2019). A review of halogenated natural products in Arctic, Subarctic and Nordic ecosystems. Emerg. Contam. 5, 89-115. doi: 10.1016/j.emcon.2019.02.007

Bommarius, A. (2015). Biocatalysis: a status report. Annu. Rev. Chem. 6, 319-345. doi: 10.1146/annurev-chembioeng-061114-1 23415

Breuer, M., Ditrich, K., Habicher, T., Hauer, B., Keseler, M. D., Sturmer, R., et al. (2004). Industrial methods for the production of optically active intermediates. Angew Chem. Int. Edit. 43, 788-824. doi: 10.1002/anie.200300599

Brokamp, A., Happe, B., and Schmidt, F. R. (1996). Cloning and nucleotide sequence of a D,L-haloalkanoic acid dehalogenase encoding gene from Alcaligenes xylosoxidans ssp. denitrificans ABIV. Biodegradation 7, 383-396. doi: $10.1007 /$ BF00056422

Bunzel, H. A., Anderson, J. L. R., and Mulholland, A. J. (2021). Designing better enzymes: insights from directed evolution. Curr. Opin. Struct. Biol. 67, 212-218. doi: $10.1016 /$ j.sbi.2020.12.015
Bustillo, A., Aleu, J., Hernández-Galán, R., and Collado, I. (2003). Biotransformation of the fungistatic compound (R)-(+)-1-(4'-chlorophenyl) propan-1-ol by Botrytis cinerea. J. Mol. Catal. B Enzym. 21, 267-271. doi: 10.1016/S1381-1177(02)00231-X

Cattoglio, C., Darzacq, X., Tjian, R., and Hansen, A. S. (2020). Estimating cellular abundances of halo-tagged proteins in live mammalian cells by flow cytometry. Bio Protoc. 10, 3527-3544. doi: 10.21769/BioProtoc.3527

Chen, B. S., and Ribeiro de Souza, F. Z. (2019). Enzymatic synthesis of enantiopure alcohols: current state and perspectives. RSC Adv. 9, 2102-2115. doi: 10.1039/C8RA09004A

Chiba, Y., Yoshida, T., Ito, N., Nishimura, H., Imada, C., Yasuda, H., et al. (2009). Isolation of a bacterium possessing a haloacid dehalogenase from a marine sediment core. Microbes Environ. 24, 276-279. doi: 10.1264/jsme2.ME09123

Chuan, D., Fan, R., Wang, Y., Ren, Y., Wang, C., Du, Y., et al. (2020). Stereocomplex poly(lactic acid)-based composite nanofiber membranes with highly dispersed hydroxyapatite for potential bone tissue engineering. Compos. Sci. Technol. 192, 1-12. doi: 10.1016/j.compscitech.2020.108107

Clayton, A. D., Labes, R., and Blacker, A. J. (2020). Combination of chemocatalysis and biocatalysis in flow. Curr. Opin. Green Sustain. Chem. 26:100378. doi: 10.1016/j.cogsc.2020.100378

de Oliveira, B. F. R., Carr, C. M., Dobson, A. D. W., and Laport, M. S. (2020). Harnessing the sponge microbiome for industrial biocatalysts. Appl. Microbiol. Biot. 104, 8131-8154. doi: 10.1007/s00253-020-10817-3

Diez, A., Prieto, M. I., Alvarez, M. J., Bautista, J. M., Garrido, A., and Puyet, A. (1996). Improved catalytic performance of a 2 -haloacid dehalogenase from Azotobacter sp. by ion-exchange immobilisation. Biochem. Biophl. Res. Co 220, 828-833. doi: 10.1006/bbrc. 1996.0489

Döbber, J., and Pohl, M. (2017). HaloTag ${ }^{\mathrm{TM}}$ : evaluation of a covalent one-step immobilization for biocatalysis. J. Biotechnol. 241, 170-174. doi: 10.1016/j.jbiotec.2016.12.004

Edbeib, M., Wahab, R., Huyop, F., Aksoy, H., and Kaya, Y. (2020). Further analysis of Burkholderia pseudomallei MF2 and identification of putative dehalogenase gene by PCR. Indones J. Chem. 20, 386-394. doi: 10.22146/ijc. 43262

Encell, L. P., Friedman Ohana, R., Zimmerman, K., Otto, P., Vidugiris, G., Wood, M. G., et al. (2012). Development of a dehalogenase-based protein fusion tag capable of rapid, selective, and covalent attachment to customizable ligands. Curr. Chem. Genomics 6, 55-71. doi: 10.2174/1875397301206010055

England, C. G., Luo, H., and Cai, W. (2015). HaloTag technology: a versatile platform for biomedical applications. Bioconjug. Chem. 26, 975-986. doi: 10.1021/acs.bioconjchem.5b00191

Erdmann, R. S., Baguley, S. W., Richens, J. H., Wissner, R. F., Xi, Z., Allgeyer, E. S., et al. (2019). Labeling strategies matter for super-resolution microscopy: a comparison between HaloTags and SNAP-tags. Cell Chem. Biol. 26, 584-592. doi: 10.1016/j.chembiol.2019.01.003

Fan, Y., Chen, S. J., Li, Q. Q., Zeng, Y., Yan, X., and Mai, B. X. (2020). Uptake of halogenated organic compounds (HOCs) into peanut and corn during the whole life cycle grown in an agricultural field. Envirorn. Pollut. 263, 1-9. doi: 10.1016/j.envpol.2020.114400

Freitas, A. I., Domingues, L., and Aguiar, T. Q. (2021). Tag-mediated singlestep purification and immobilization of recombinant proteins toward proteinengineered advanced materials. J. Adv. Res. doi: 10.1016/j.jare.2021.06.010. [Epub ahead of print].

Garbujo, S., Galbiati, E., Salvioni, L., Mazzucchelli, M., Frascotti, G., Sun, X., et al. (2020). Functionalization of colloidal nanoparticles with a discrete number of ligands based on a "HALO-bioclick" reaction. Chem. Commun. 56, 11398-11401. doi: 10.1039/D0CC04355A

Gong, Y., Ma, S., Wang, Y., Xu, Y., Sun, A., Zhang, Y., et al. (2018). Characterization of a novel deep-sea microbial esterase EstC10 and its use in the generation of (R)-methyl-2-chloropropionate. J. Oceanol. Limnol. 36, 473-482. doi: 10.1007/s00343-018-6297-4

Gul, I., Bogale, T. F., Chen, Y., Yang, X., Fang, R., Feng, J., et al. (2020a). A paper-based whole-cell screening assay for directed evolutiondriven enzyme engineering. Appl. Microbiol. Biot. 104, 6013-6022. doi: $10.1007 / \mathrm{s} 00253-020-10615-\mathrm{x}$

Gul, I., Wang, Q., Jiang, Q., Fang, R., and Tang, L. (2020b). Enzyme immobilization on glass fiber membrane for detection of halogenated compounds. Anal. Biochem. 609, 1-8. doi: 10.1016/j.ab.2020.113971 
Gurushankara, H. P. (2021). "Chapter 13-Recent developments in biocatalysis and its influence on the pharmaceutical industry," in Recent Developments in Applied Microbiology and Biochemistry, ed B. Viswanath (Cambridge, MA: Academic Press), 127-139.

Hamid, A. A. A., Hamid, T. H. T. A., Wahab, R. A., Omar, M. S. S., and Huyop, F. (2015). An S188V mutation alters substrate specificity of non-stereospecific $\alpha$-haloalkanoic acid dehalogenase E (DehE). PLoS ONE 10:e0121687. doi: 10.1371/journal.pone.0121687

Hamid, T. H. T. A., Hamid, A. A. A., and Huyop, F. (2011). A review on non-stereospecific haloalkanoic acid dehalogenases. Afr. J. Biotechnol. 10, 9725-9736. doi: 10.5897/AJB11.934

Hasan, A. K. M. Q., Takada, H., Koshikawa, H., Liu, J. Q., Kurihara, T., Esaki, N., et al. (1994). Two kinds of 2-halo acid dehalogenases from Pseudomonas sp. YL induced by 2-chloroacrylate and 2-chloropropionate. Biosci. Biotechnol. Biochem. 58, 1599-1602. doi: 10.1271/bbb.58.1599

Heidarrezaei, M., Shokravi, H., Huyop, F., Rahimian Koloor, S. S., and Petru, M. (2020). Isolation and characterization of a novel bacterium from the marine environment for trichloroacetic acid bioremediation. Appl. Sci. 10, 1-17. doi: 10.3390/app10134593

Hellal, J., Joulian, C., Urien, C., Ferreira, S., Denonfoux, J., Hermon, L., et al. (2021). Chlorinated ethene biodegradation and associated bacterial taxa in multi-polluted groundwater: insights from biomolecular markers and stable isotope analysis. Sci. Total Environ. 763, 1-10. doi: 10.1016/j.scitotenv.2020.142950

Hermon, L., Denonfoux, J., Hellal, J., Joulian, C., Ferreira, S., Vuilleumier, S., et al. (2018). Dichloromethane biodegradation in multi-contaminated groundwater: insights from biomolecular and compound-specific isotope analyses. Water Res. 142, 217-226. doi: 10.1016/j.watres.2018.05.057

Higgins, T. P., Hope, S. J., Effendi, A. J., Dawson, S., and Dancer, B. N. (2005). Biochemical and molecular characterisation of the 2,3-dichloro1-propanol dehalogenase and stereospecific haloalkanoic dehalogenases from a versatile Agrobacterium sp. Biodegradation 16, 485-492. doi: 10.1007/s10532-004-5670-5

Hill, K. E., Marchesi, J. R., and Weightman, A. J. (1999). Investigation of two evolutionarily unrelated halocarboxylic acid dehalogenase gene families. J. Bacteriol. 181, 2535-2547. doi: 10.1128/JB.181.8.2535-2547.1999

Hisano, T., Hata, Y., Fujii, T., Liu, J.-Q., Kurihara, T., Esaki, N., et al. (1996). Crystal structure of L-2-haloacid dehalogenase from Pseudomonas sp. YL: an $\alpha / \beta$ hydrolase structure that is different from the $\alpha / \beta$ hydrolase fold. J. Biol. Chem. 271, 20322-20330. doi: 10.1074/jbc.271.34.20322

Horisaki, T., Yoshida, E., Sumiya, K., Takemura, T., Yamane, H., and Nojiri, H. (2011). Isolation and characterization of monochloroacetic acid-degrading bacteria. J. Gen. Appl. Microbiol. 57, 277-284. doi: 10.2323/jgam.57.277

Huyop, F., and Sudi, I. Y. (2012). D-specific dehalogenases, a review. Biotechnol. Biotec. Eq. 26, 2817-2822. doi: 10.5504/BBEQ.2011.0143

Idris and Ratnaningsih, E. (2015). Cloning of haloacid dehalogenase gene from Bacillus cereus local strain with the addition of restriction sites. Procedia Chem. 16, 314-320. doi: 10.1016/j.proche.2015.12.058

Janssen, D. B. (2004). Evolving haloalkane dehalogenases. Curr. Opin. Chem. Biol. 8, 150-159. doi: 10.1016/j.cbpa.2004.02.012

Kajla, S., Nagi, G. K., and Kumari, R. (2021). Microorganisms employed in the removal of contaminants from wastewater of iron and steel industries. Rend Lincei Sci. Fis. 32, 257-272. doi: 10.1007/s12210-021-00982-6

Kim, D.-H., Park, C. G., and Kim, Y. J. (2020). Characterizing the potential estrogenic and androgenic activities of two disinfection byproducts, monohaloacetic acids and haloacetamides, using in vitro bioassays. Chemosphere 242, 1-6. doi: 10.1016/j.chemosphere.2019.125198

Kirkinci, S. F., Edbeib, M. F., Aksoy, H. M., Marakli, S., and Kaya, Y. (2021). Identification of Dalapon degrading bacterial strain, Psychrobacter sp. TaeBurcu001 isolated from Antarctica. Polar Sci. 28, 1-9. doi: 10.1016/j.polar.2021.100656

Köhler, R., Brokamp, A., Schwarze, R., Reiting, R. H., and Schmidt, F. R. (1998). Characteristics and DNA-sequence of a cryptic haloalkanoic acid dehalogenase from Agrobacterium tumefaciens RS5. Curr. Microbiol. 36, 96-101. doi: 10.1007/s002849900286

Kondo, H., Fujimoto, K. J., Tanaka, S., Deki, H., and Nakamura, T. (2015). Theoretical prediction and experimental verification on enantioselectivity of haloacid dehalogenase L-DEX YL with chloropropionate. Chem. Phys. Lett. 623, 101-107. doi: 10.1016/j.cplett.2015. 01.053

Kondo, H., Nakamura, T., and Tanaka, S. (2014). A significant role of Arg41 residue in the enzymatic reaction of haloacid dehalogenase L-DEX YL studied by QM/MM method. J. Mol. Catal. B Enzym. 110, 23-31. doi: 10.1016/j.molcatb.2014.09.006

Kumar, A., Pillay, B., and Olaniran, A. O. (2016). L-2-haloacid dehalogenase from Ancylobacter aquaticus UV5: sequence determination and structure prediction. Int. J. Biol. Macromol. 83, 216-225. doi: 10.1016/j.ijbiomac.2015.11.066

Kunka, A., Damborsky, J., and Prokop, Z. (2018). "Chapter Seven - Haloalkane Dehalogenases From Marine Organisms," in Methods in Enzymology, ed B. S. Moore (Cambridge, MA: Academic Press), 203-251.

Kurade, M. B., Ha, Y. H., Xiong, J. Q., Govindwar, S. P., Jang, M., and Jeon, B. H. (2021). Phytoremediation as a green biotechnology tool for emerging environmental pollution: a step forward towards sustainable rehabilitation of the environment. Chem. Eng. J. 415, 1-19. doi: 10.1016/j.cej.2021.129040

Kurihara, T. (2011). A mechanistic analysis of enzymatic degradation of organohalogen compounds. Biosci. Biotechnol. Biochem. 75, 189-198. doi: $10.1271 /$ bbb. 100746

Kurihara, T., and Esaki, N. (2008). Bacterial hydrolytic dehalogenases and related enzymes: occurrences, reaction mechanisms, and applications. Chem. Rec. 8, 67-74. doi: $10.1002 /$ tcr.20141

Kurihara, T., Esaki, N., and Soda, K. (2000). Bacterial 2-haloacid dehalogenases: structures and reaction mechanisms. J. Mol. Catal. B Enzym. 10, 57-65. doi: 10.1016/S1381-1177(00)00108-9

Kurumbang, N. P., Dvorak, P., Bendl, J., Brezovsky, J., Prokop, Z., and Damborsky, J. (2014). Computer-assisted engineering of the synthetic pathway for biodegradation of a toxic persistent pollutant. ACS Synth. Biol. 3, 172-181. doi: $10.1021 / \mathrm{sb} 400147 \mathrm{n}$

Leemans Martin, L., Peschke, T., Venturoni, F., and Mostarda, S. (2020). Pharmaceutical industry perspectives on flow chemocatalysis and biocatalysis. Curr. Opin. Green Sustain 25:100350. doi: 10.1016/j.cogsc.2020.04.011

Leigh, J. A., Skinner, A. J., and Cooper, R. A. (1986). Isolation and partial characterisation of dehalogenase-deficient mutants of a Rhizobium sp.. FEMS Microbiol. Lett. 36, 163-166. doi: 10.1111/j.1574-6968.1986.tb01688.x

Li, J., Cai, W., and Zhu, L. (2011). The characteristics and enzyme activities of 4-chlorophenol biodegradation by Fusarium sp. Bioresource Technol. 102, 2985-2989. doi: 10.1016/j.biortech.2010.10.006

Liao, L., Sun, X., Zeng, Y., Luo, W., Yu, Y., and Chen, B. (2015). A new Lhaloacid dehalogenase from the Arctic psychrotrophic Pseudoalteromonas sp. BSW20308. Polar Biol. 38, 1161-1169. doi: 10.1007/s00300-015-1674-3

Liu, J., Zhai, H., Sun, Y., Wu, S., and Chen, S. (2021). Developing high strength poly(L-lactic acid) nanofiber yarns for biomedical textile materials: a comparative study of novel nanofiber yarns and traditional microfiber yarns. Mater. Lett. 300:130229. doi: 10.1016/j.matlet.2021.130229

Liu, J. Q., Kurihara, T., Hasan, A. K., Nardi-Dei, V., Koshikawa, H., Esaki, N., et al. (1994). Purification and characterization of thermostable and nonthermostable 2-haloacid dehalogenases with different stereospecificities from Pseudomonas sp. strain YL. Appl. Environ. Microbiol. 60, 2389-2393. doi: 10.1128/aem.60.7.2389-2393.1994

Long, K., Sha, Y., Mo, Y., Wei, S., Wu, H., Lu, D., et al. (2021). Androgenic and teratogenic effects of iodoacetic acid drinking water disinfection byproduct in vitro and in vivo. Environ. Sci. Technol. 55, 3827-3835. doi: 10.1021 /acs.est.0c06620

Longo, L. M., Kumru, O. S., Middaugh, C. R., and Blaber, M. (2014). Evolution and design of protein structure by folding nucleus symmetric expansion. Structure 22, 1377-1384. doi: 10.1016/j.str.2014.08.008

Lou, J., Wang, W., and Zhu, L. (2021). Transformation of emerging disinfection byproducts Halobenzoquinones to haloacetic acids during chlorination of drinking water. Chem. Eng. J. 418, 129326. doi: 10.1016/j.cej.2021. 129326

Lou, Y. Y., Fontmorin, J.-M., Amrane, A., Fourcade, F., and Geneste, F. (2021). Metallic nanoparticles for electrocatalytic reduction of halogenated organic compounds: A review. Electrochim. Acta 377, 138039. doi: 10.1016/j.electacta.2021.138039

Ma, F., He, L., Lindner, E., and Wu, D. Y. (2021). Highly porous poly(L-lactic) acid nanofibers as a dual-signal paper-based bioassay platform for in vitro diagnostics. Appl. Surf. Sci. 542:148732. doi: 10.1016/j.apsusc.2020.148732 
Marshall, J. R., Mangas-Sanchez, J., and Turner, N. J. (2021). Expanding the synthetic scope of biocatalysis by enzyme discovery and protein engineering. Tetrahedron 82:131926. doi: 10.1016/j.tet.2021.131926

Mazur, A., Prudnikova, T., Grinkevich, P., Mesters, J. R., Mrazova, D., Chaloupkova, R., et al. (2021). The tetrameric structure of the novel haloalkane dehalogenase DpaA from Paraglaciecola agarilytica $\mathrm{NO}_{2}$. Acta Crystallogr. D Struct. Biol. 77, 347-356. doi: 10.1107/S2059798321000486

Mena-Benitez, G. L., Gandia-Herrero, F., Graham, S., Larson, T. R., McQueenMason, S. J., French, C. E., et al. (2008). Engineering a catabolic pathway in plants for the degradation of 1,2-dichloroethane. Plant Physiol. 147, 1192-1198. doi: 10.1104/pp.108.119008

Merrill, R. A., Song, J., Kephart, R. A., Klomp, A. J., Noack, C. E., and Strack, S. (2019). A robust and economical pulse-chase protocol to measure the turnover of HaloTag fusion proteins. J. Biol. Chem. 294, 16164-16171. doi: $10.1074 /$ jbc.RA119.010596

Minner-Meinen, R., Weber, J. N., Albrecht, A., Matis, R., Behnecke, M., Tietge, C., et al. (2021). Split-HaloTag $®$ imaging assay for sophisticated microscopy of protein-protein interactions in planta. Plant Commun. 2:100212. doi: 10.1016/j.xplc.2021.100212

Mohamed, E., Mohamed, J., Huyop, F., Wahab, R., and Abdul-Hamid, H. (2020). Developing herbicide tolerant transgenic plants for sustainable weed management. Int. J. Emerg. Trends Eng. Res. 8, 60-66. doi: $10.30534 /$ ijeter/2020/0981.22020

Muzikár, M., Kresinová, Z., Svobodová, K., Filipová, A., Cvančarová, M., Cajthamlová, K., et al. (2011). Biodegradation of chlorobenzoic acids by ligninolytic fungi. J. Hazard. Mater. 196, 386-394. doi: 10.1016/j.jhazmat.2011.09.041

Narayanan, H., Dingfelder, F., Butt,é, A., Lorenzen, N., Sokolov, M., and Arosio, P. (2021). Machine Learning for biologics: opportunities for protein engineering, developability, and formulation. Trends Pharmacol. Sci. 42, 151-165. doi: 10.1016/j.tips.2020.12.004

Nardi-Dei, V., Kurihara, T., Park, C., Miyagi, M., Tsunasawa, S., Soda, K., et al. (1999). DL-2-Haloacid dehalogenase from Pseudomonas sp. 113 is a new class of dehalogenase catalyzing hydrolytic dehalogenation not involving enzyme-substrate ester intermediate. J. Biol. Chem. 274, 20977-20981. doi: $10.1074 /$ jbc.274.30.20977

Nguyen, B. T., Hsieh, J. L., Lo, S. C., Wang, S. Y., Hung, C. H., Huang, E., et al. (2021). Biodegradation of dioxins by Burkholderia cenocepacia strain 869T2: role of 2-haloacid dehalogenase. J. Hazard. Mater. 401:123347. doi: $10.1016 /$ j.jhazmat.2020.123347

Novak, H., and Littlechild, J. (2013). "Marine enzymes with applications for biosynthesis of fine chemicals," in Marine Enzymes for Biocatalysis, ed A. Trincone (Sawston: Woodhead Publishing), 89-106.

Novak, H. R., Sayer, C., Isupov, M. N., Paszkiewicz, K., Gotz, D., Spragg, A. M., et al. (2013a). Marine Rhodobacteraceae L-haloacid dehalogenase contains a novel His/Glu dyad that could activate the catalytic water. FEBS J. 280, 1664-1680. doi: $10.1111 /$ febs.12177

Novak, H. R., Sayer, C., Panning, J., and Littlechild, J. A. (2013b). Characterisation of an L-haloacid dehalogenase from the marine psychrophile Psychromonas ingrahamii with potential industrial application. Mar. Biotechnol. 15, 695-705. doi: $10.1007 / \mathrm{s} 10126-013-9522-3$

Oyewusi, H. A., Huyop, F., and Wahab, R. A. (2020a). Molecular docking and molecular dynamics simulation of Bacillus thuringiensis dehalogenase against haloacids, haloacetates and chlorpyrifos. J. Biomol. Struct. Dyn. 38, 1-16. doi: 10.1080/07391102.2019.1580616

Oyewusi, H. A., Huyop, F., Wahab, R. A., and Hamid, A. A. A. (2021a). In silico assessment of dehalogenase from Bacillus thuringiensis $\mathrm{H} 2$ in relation to its salinity-stability and pollutants degradation. J. Biomol. Struct. Dyn. 39, 1-15. doi: 10.1080/07391102.2021.1927846

Oyewusi, H. A., Wahab, R. A., and Huyop, F. (2020b). Dehalogenase-producing halophiles and their potential role in bioremediation. Mar. Pollut. Bull. 160:111603. doi: 10.1016/j.marpolbul.2020.111603

Oyewusi, H. A., Wahab, R. A., and Huyop, F. (2021b). Whole genome strategies and bioremediation insight into dehalogenase-producing bacteria. Mol. Biol. Rep. 48, 2687-2701. doi: 10.1007/s11033-021-06239-7

Park, C., Kurihara, T., Yoshimura, T., Soda, K., and Esaki, N. (2003). A new DL-2-haloacid dehalogenase acting on 2-haloacid amides: purification, characterization, and mechanism. J. Mol. Catal. B Enzym. 23, 329-336. doi: 10.1016/S1381-1177(03)00096-1
Parker, K., and Colby, J. (1995). Immobilisation of the D-2-haloacid dehalogenase from Pseudomonas putida strain AJ1/23. Biodegradation 6, 191-201. doi: 10.1007/BF00700457

Poelarends, G. J., and Whitman, C. P. (2010). "Mechanistic and structural studies of microbial dehalogenases: How nature cleaves a carbon-halogen bond," in Comprehensive Natural Products II, ed H. W. Liu and L. Mander (Oxford: Elsevier), 89-123.

Polnisch, E., Kneifel, H., Franzke, H., and Hofmann, K. H. (1991). Degradation and dehalogenation of monochlorophenols by the phenol-assimilating yeast Candida maltosa. Biodegradation 2, 193-199. doi: 10.1007/BF00124493

Ratnaningsih, E., and Idris, I. (2018). Cloning and expression of haloacid dehalogenase gene from Bacillus cereus IndB1. Indones. J. Biotechnol. 22, 55-60. doi: 10.22146/ijbiotech. 27338

Ridder, I. S., Rozeboom, H. J., Kalk, K. H., Janssen, D. B., and Dijkstra, B. W. (1997). Three-dimensional structure of L-2-haloacid dehalogenase from Xanthobacter autotrophicus GJ10 complexed with the substrate-analogue formate. J. Biol. Chem. 272, 33015-33022. doi: 10.1074/jbc.272.52.33015

Rosland Abel, S., Ibrahim, N., and Huyop, F. (2012). Identification of Serratia marcescens SE1 and determination of its herbicide 2,2-dichloropropionate (2,2-DCP) degradation potential. Malays. J. Microbiol. 8, 259-265. doi: $10.21161 / \mathrm{mjm} .44412$

Rye, C. A., Isupov, M. N., Lebedev, A. A., and Littlechild, J. A. (2009). Biochemical and structural studies of a L-haloacid dehalogenase from the thermophilic archaeon Sulfolobus tokodaii. Extremophiles 13, 179-190. doi: $10.1007 /$ s00792-008-0208-0

Santi, M., Sancineto, L., Nascimento, V., Braun Azeredo, J., Orozco, E. V. M., Andrade, L. H., et al. (2021). Flow biocatalysis: a challenging alternative for the synthesis of apis and natural compounds. Int. J. Mol. Sci. 22:990. doi: 10.3390/ijms22030990

Satpathy, R., Konkimalla, V. S. B., and Ratha, J. (2015). In-silico gene co-expression network analysis in Paracoccidioides brasiliensis with reference to haloacid dehalogenase superfamily hydrolase gene. J. Pharm. Bioallied Sci. 7, 212-217. doi: 10.4103/0975-7406.160023

Satpathy, R., Konkimalla, V. S. B., and Ratha, J. (2016). In silico phylogenetic analysis and molecular modelling study of 2-haloalkanoic acid dehalogenase enzymes from bacterial and fungal origin. Adv. Bioinform. 2016, 8701201-8701201. doi: 10.1155/2016/8701201

Schmidberger, J. W., Wilce, J. A., Tsang, J. S. H., and Wilce, M. C. J. (2007). Crystal structures of the substrate free-enzyme, and reaction intermediate of the HAD superfamily member, haloacid dehalogenase DehIVa from Burkholderia cepacia MBA4. J. Mol. Biol. 368, 706-717. doi: 10.1016/j.jmb.2007.02.015

Schmidberger, J. W., Wilce, J. A., Weightman, A. J., Whisstock, J. C., and Wilce, M. C. (2008). The crystal structure of DehI reveals a new alpha-haloacid dehalogenase fold and active-site mechanism. J. Mol. Biol. 378, 284-294. doi: $10.1016 /$ j.jmb.2008.02.035

Schober, M., and Faber, K. (2013). Inverting hydrolases and their use in enantioconvergent biotransformations. Trends Biotechnol. 31, 468-478. doi: 10.1016/j.tibtech.2013.05.005

Sengupta, S., Das, P., and Datta, S. (2020). "Fermentative production of optically pure lactic acid from renewable materials," in Encyclopedia of Renewable and Sustainable Materials, ed S. Hashmi and I. A. Choudhury (Oxford: Elsevier), 447-453.

Shahar, H., Tan, L. L., Ta, G. C., and Heng, L. Y. (2019a). Detection of halogenated hydrocarbon pollutants using enzymatic reflectance biosensor. Sensors Actuat. B Chem. 281, 80-89. doi: 10.1016/j.snb.2018.10.076

Shahar, H., Tan, L. L., Ta, G. C., and Heng, L. Y. (2019b). Optical enzymatic biosensor membrane for rapid in situ detection of organohalide in water samples. Microchem. J. 146, 41-48. doi: 10.1016/j.microc.2018. 12.052

Siwek, A., Omi, R., Hirotsu, K., Jitsumori, K., Esaki, N., Kurihara, T., et al. (2013). Binding modes of DL-2-haloacid dehalogenase revealed by crystallography, modeling and isotope effects studies. Arch. Biochem. Biophys. 540, 26-32. doi: 10.1016/j.abb.2013.09.012

Smith, J. M., Harrison, K., and Colby, J. (1990). Purification and characterization of D-2-haloacid dehalogenase from Pseudomonas putida strain AJ1/23. J. Gen. Microbiol. 136, 881-886. doi: 10.1099/00221287-136-5-881

Soda, K., Kurihara, T., Liu, J. Q., NardiDei, V., Park, C., Miyagi, M., et al. (1996). Bacterial 2-haloacid dehalogenases: structures and catalytic properties. Pure Appl. Chem. 68, 2097-2103. doi: 10.1351/pac199668112097 
Su, X., Deng, L., Kong, K. F., and Tsang, J. S. (2013). Enhanced degradation of haloacid by heterologous expression in related Burkholderia species. Biotechnol. Bioeng. 110, 2687-2696. doi: 10.1002/bit.24917

Sudi, I. Y., Hamid, A. A., Shamsir, M. S., Jamaluddin, H., Wahab, R. A., and Huyop, F. (2014). Insights into the stereospecificity of the D-specific dehalogenase from Rhizobium sp. RC1 toward D- and L-2-chloropropionate. Biotechnol. Biotechnol. Equip. 28, 608-615. doi: 10.1080/13102818.2014.937907

Tanokura, M., Miyakawa, T., Guan, L., and Hou, F. (2015). Structural analysis of enzymes used for bioindustry and bioremediation. Biosci. Biotech. Biochem. 79, 1391-1401. doi: 10.1080/09168451.2015.1052770

Taylor Stephen, C. (1985). D-2-haloalkanoic acid halidohydrolase. US19850789765. U.S. Patent.

Thippeswamy, M., Rajasrerlatha, V., Shubha, D., and Niveditha, B. T. (2021). "Chapter 24- Metagenomics and future perspectives in discovering pollutant degrading enzymes from soil microbial communities," in Recent Developments in Applied Microbiology and Biochemistry, ed B. Viswanath (Cambridge, MA: Academic Press), 257-267.

Tunyasuvunakool, K., Adler, J., Wu, Z., Green, T., Zielinski, M., Žídek, A., et al. (2021). Highly accurate protein structure prediction for the human proteome. Nature 596, 1-9. doi: 10.1038/s41586-021-03828-1

van der Ploeg, J., van Hall, G., and Janssen, D. B. (1991). Characterization of the haloacid dehalogenase from Xanthobacter autotrophicus GJ10 and sequencing of the dhlB gene. J. Bacteriol. 173, 7925-7933. doi: 10.1128/jb.173.24.7925-7933.1991

Vrancken, J. P. M., Tame, J. R. H., and Voet, A. R. D. (2020). Development and applications of artificial symmetrical proteins. Comput. Struct. Biotechnol. J. 18, 3959-3968. doi: 10.1016/j.csbj.2020.10.040

Wahhab, B., Anuar, N., Wahab, R., Al-Nimer, M., Samsulrizal, N., Abdul Hamid, A., et al. (2020). Characterization of a 2,2-dichloropropionic acid (2,2-DCP) degrading alkalotorelant Bacillus megaterium strain BHS1 isolated from blue lake in turkey. J. Trop. Life Sci. 10, 245-252. doi: 10.11594/jtls.10.03.08

Wang, C., Xi, J., Hu, H. Y., and Wen, X. (2009). Biodegradation of gaseous chlorobenzene by white-rot fungus Phanerochaete chrysosporium. Biomed. Environ. Sci. 21, 474-478. doi: 10.1016/S0895-3988(09)60005-2

Wang, S., Cheng, Z., Xu, Y., Yang, L., Wang, J. B., Tian, Z., et al. (2020). Structure-guided protein design of fluoroacetate dehalogenase for kinetic resolution of rac-2-bromobutyric acid. Green Synth. Catal. 1, 60-65. doi: 10.1016/j.gresc.2020.05.004

Wang, Y., Cao, X., Feng, Y., and Xue, S. (2016). Environment-induced conformational and functional changes of L-2-haloacid dehalogenase. J. Biosci. Bioeng. 121, 491-496. doi: 10.1016/j.jbiosc.2015.09.008

Wang, Y., Feng, Y., Cao, X., Liu, Y., and Xue, S. (2018). Insights into the molecular mechanism of dehalogenation catalyzed by D-2-haloacid dehalogenase from crystal structures. Sci. Rep. 8:1454. doi: 10.1038/s41598-017-19050-x

Wang, Y., Xue, S., Zhou, Q., and Pei, D. (2020). Recent progress in 2-haloacid dehalogenases. Chin. J. Biotechnol. 36, 868-878. doi: 10.13345/j.cjb.190370

Watanabe, S., Ito, M., and Kigawa, T. (2021). DiRect: Site-directed mutagenesis method for protein engineering by rational design. Biochem. Biophys. Res. Co 551, 107-113. doi: 10.1016/j.bbrc.2021.03.021

Weightman, A. J., Weightman, A. L., and Slater, J. H. (1982). Stereospecificity of 2-monochloropropionate dehalogenation by the two dehalogenases of Pseudomonas putida PP3: evidence for two different dehalogenation mechanisms. Microbiology 128, 1755-1762. doi: 10.1099/00221287-128-8-1755

Woolfson, D. N. (2021). A brief history of de novo protein design: minimal, rational, and computational. J. Mol. Biol. doi: 10.1016/j.jmb.2021.167160. [Epub ahead of print].

Wu, L., Qin, L., Nie, Y., Xu, Y., and Zhao, Y.-L. (2021). Computer-aided understanding and engineering of enzymatic selectivity. Biotechnol. Adv. doi: 10.1016/j.biotechadv.2021.107793. [Epub ahead of print].

Wu, Q., Bouwman, H., Uren, R. C., van der Lingen, C. D., and Vetter, W. (2019). Halogenated natural products and anthropogenic persistent organic pollutants in chokka squid (Loligo reynaudii) from three sites along the South Atlantic and Indian Ocean coasts of South Africa. Environ. Pollut. 255:113282. doi: 10.1016/j.envpol.2019.113282

Xie, G., Pan, D., He, W., and Gao, G. (2015). Application of L-2-haloacid dehalogenase from thermophilic Archaea Sulfolobus tokodaii in the production of D-lactic acid. Chem. J. Chinese Univ. 36, 698-703. doi: 10.7503/cjcu20140739
Xiong, W., Liu, B., Shen, Y., Jing, K., and Savage, T. R. (2021). Protein engineering design from directed evolution to de novo synthesis. Biochem. Eng. J. 174:108096. doi: 10.1016/j.bej.2021.108096

Xu, S., Qin, S., and Pan, X.-M. (2004). Thermal and conformational stability of Ssh10b protein from archaeon Sulfolobus shibattae. Biochem. J. 382, 433-440. doi: $10.1042 / B J 20040191$

Yavari Maroufi, L., Ghorbani, M., Mohammadi, M., and Pezeshki, A. (2021). Improvement of the physico-mechanical properties of antibacterial electrospun poly lactic acid nanofibers by incorporation of guar gum and thyme essential oil. Colloid Surface A 622:126659. doi: 10.1016/j.colsurfa.2021.126659

Yu, F., Li, Y., Wang, H., Peng, T., Wu, Y. R., and Hu, Z. (2021). Microbial debromination of hexabromocyclododecanes. Appl. Microbiol. Biot. 105, 4535-4550. doi: 10.1007/s00253-021-11095-3

Zainal Abidin, M. H., Abd Halim, K. B., Huyop, F., Tengku Abdul Hamid, T. H., Abdul Wahab, R., and Abdul Hamid, A. A. (2019). The mechanistic role of active site residues in non-stereo haloacid dehalogenase E (DehE). J. Mol. Graph. Model. 90, 219-225. doi: 10.1016/j.jmgm.2019.05.003

Zakary, S., Oyewusi, H., and Huyop, F. (2021). Genomic analysis of mesorhizobium loti strain tono reveals dehalogenases for bioremediation. $J$. Trop. Life Sci. 11, 67-77. doi: 10.11594/jtls.11.01.09

Zhang, C., Allen, K. N., and Dunawaymariano, D. (2018). Mechanism of substrate recognition and catalysis of the haloalkanoic acid dehalogenase family member $\alpha$-phosphoglucomutase. Biochemistry 57, 4504-4517. doi: 10.1021/acs.biochem.8b00396

Zhang, C., Yuan, Z., Liu, Y., Zhang, Q., Chen, Y., Saleem, S., et al. (2021). The online detection of halogenated hydrocarbon in the atmosphere. Opt Laser Eng 142:106586. doi: 10.1016/j.optlaseng.2021.106586

Zhang, J., Cao, X., Xin, Y., Xue, S., and Zhang, W. (2013). Purification and characterization of a dehalogenase from Pseudomonas stutzeri DEH130 isolated from the marine sponge Hymeniacidon perlevis. World J. Microbiiol. Biotechnol. 29, 1791-1799. doi: 10.1007/s11274-013-1340-2

Zhang, J., Jiang, L., Chen, X., Lv, K., Basiony, M., Zhu, G., et al. (2021). Recent advances in biotechnology for marine enzymes and molecules. Curr. Opin. Biotechnol. 69, 308-315. doi: 10.1016/j.copbio.2021.05.009

Zhang, J., Xin, Y., Cao, X., Xue, S., and Zhang, W. (2014). Purification and characterization of 2-haloacid dehalogenase from marine bacterium Paracoccus sp. DEH99, isolated from marine sponge Hymeniacidon perlevis. J. Ocean Univ. China 13, 91-96. doi: 10.1007/s11802-014-2357-3

Zhang, M., Shi, Q., Song, X., Wang, H., and Bian, Z. (2019). Recent electrochemical methods in electrochemical degradation of halogenated organics: a review. Environ. Sci. Pollut. Res. Int. 26, 10457-10486. doi: 10.1007/s11356-019-04533-3

Zhou, H., Li, Y., Jiang, R., Wang, X., Wang, Y., Xue, Y., et al. (2021). An efficient route towards R-2-phenoxypropionic acid synthesis for biotransformative production of R-2-(4-hydroxyphenoxy) propionic acid. Chinese J. Chem. Eng. 32, 315-323. doi: 10.1016/j.cjche.2020.06.013

Conflict of Interest: JX is an employee of Zhengzhou Tuoyang Industrial Co., Ltd.

The remaining authors declare that the research was conducted in the absence of any commercial or financial relationships that could be construed as a potential conflict of interest.

Publisher's Note: All claims expressed in this article are solely those of the authors and do not necessarily represent those of their affiliated organizations, or those of the publisher, the editors and the reviewers. Any product that may be evaluated in this article, or claim that may be made by its manufacturer, is not guaranteed or endorsed by the publisher.

Copyright $\odot 2021$ Wang, Xiang, Zhou, Xu and Pei. This is an open-access article distributed under the terms of the Creative Commons Attribution License (CC BY). The use, distribution or reproduction in other forums is permitted, provided the original author(s) and the copyright owner(s) are credited and that the original publication in this journal is cited, in accordance with accepted academic practice. No use, distribution or reproduction is permitted which does not comply with these terms. 